\title{
ACR Guidance Document on MR Safe Practices: 2013
}

\author{
Expert Panel on MR Safety: Emanuel Kanal, MD, ${ }^{1 *}$ A. James Barkovich, MD, ${ }^{2}$ \\ Charlotte Bell, MD, ${ }^{3}$ James P. Borgstede, MD, ${ }^{4}$ William G. Bradley Jr, MD, PhD, ${ }^{5}$ \\ Jerry W. Froelich, MD, ${ }^{6}$ J. Rod Gimbel, MD, ${ }^{7}$ John W. Gosbee, MD, ${ }^{8}$ \\ Ellisa Kuhni-Kaminski, RT, ${ }^{1}$ Paul A. Larson, MD, ${ }^{9}$ James W. Lester Jr, MD, ${ }^{10}$ \\ John Nyenhuis, PhD, ${ }^{11}$ Daniel Joe Schaefer, PhD, ${ }^{12}$ Elizabeth A. Sebek, RN, BSN, ${ }^{1}$ \\ Jeffrey Weinreb, MD, ${ }^{13}$ Bruce L. Wilkoff, MD, ${ }^{14}$ Terry O. Woods, PhD, ${ }^{15}$ \\ Leonard Lucey, JD, ${ }^{16}$ and Dina Hernandez, BSRT ${ }^{16}$
}

Because there are many potential risks in the MR environment and reports of adverse incidents involving patients, equipment and personnel, the need for a guidance document on MR safe practices emerged. Initially published in 2002, the ACR MR Safe Practices Guidelines established de facto industry standards for safe and responsible practices in clinical and research MR environments. As the MR industry changes the document is reviewed, modified and updated. The most recent version will reflect these changes.

Key Words: MR safety; MR; MR safe practices

J. Magn. Reson. Imaging 2013;37:501-530.

(c) 2013 Wiley Periodicals, Inc.

${ }^{1}$ Department of Radiology, University of Pittsburgh Medical Center, Pittsburgh, Pennsylvania, USA.

${ }^{2}$ Department of Radiology and Biomedical Imaging, University of California, San Francisco, California, USA.

${ }^{3}$ Milford Anesthesia Associates, Milford, Connecticut, USA.

${ }^{4}$ University of Colorado, Denver, Colorado, USA.

${ }^{5}$ Department of Radiology, University of California San Diego Medical Center, San Diego, California, USA.

${ }^{6}$ Department of Radiology, University of Minnesota, Minneapolis, Minnesota, USA.

${ }^{7}$ Cardiology Associates of E. Tennessee, Knoxville, Tennessee, USA.

${ }^{8}$ University of Michigan Health System and Red Forest Consulting LLC, Ann Arbor, Michigan, USA.

${ }^{9}$ Radiology Associates of the Fox Valley, Neenah, Wisconsin, USA.

${ }^{10}$ Durham Radiology Associates, Raleigh, North Carolina, USA.

${ }^{11}$ Department of Electrical and Computer Engineering, Purdue University, West Lafayette, Indiana, USA.

${ }^{12}$ MR Systems Engineering, GE Healthcare, Waukesha, Wisconsin, USA.

${ }^{13}$ Yale School of Medicine, New Haven, Connecticut, USA.

${ }^{14}$ Cleveland Clinic, Cleveland, Ohio, USA.

${ }^{15}$ FDA Center for Devices \& Radiological Health, Silver Spring, Maryland, USA.

${ }^{16}$ American College of Radiology, Reston, Virginia, USA.

Reprint requests to: Department of Quality \& Safety, American College of Radiology, 1891 Preston White Drive, Reston, VA 20191-4397.

*Address reprint requests to: E.K., University of Pittsburgh Medical Center, Presbyterian University Hospital\Presbyterian South Tower, Room 4776, Pittsburgh, PA 15213. E-mail: ekanal@pitt.edu

Received October 3, 2012; Accepted December 4, 2012.

DOI 10.1002/jmri.24011

View this article online at wileyonlinelibrary.com.

(C) 2013 Wiley Periodicals, Inc.
THERE ARE POTENTIAL risks in the MR environment, not only for the patient $(1,2)$ but also for the accompanying family members, attending health care professionals, and others who find themselves only occasionally or rarely in the magnetic fields of MR scanners, such as security or housekeeping personnel, firefighters, police, etc. (3-6). There have been reports in the medical literature and print-media detailing Magnetic Resonance Imaging (MRI) adverse incidents involving patients, equipment and personnel that spotlighted the need for a safety review by an expert panel. To this end, the American College of Radiology originally formed the Blue Ribbon Panel on MR Safety. First constituted in 2001, the panel was charged with reviewing existing MR safe practices and guidelines (5-8) and issuing new ones as appropriate for MR examinations. Published initially in 2002 (4), the ACR MR Safe Practice Guidelines established de facto industry standards for safe and responsible practices in clinical and research MR environments. These were subsequently reviewed and updated in May of 2004 (3). After reviewing substantial feedback from the field and installed base, as well as changes that had transpired throughout the MR industry since the publication of the 2004 version of this document, the panel extensively reviewed, modified, and updated the entire document in 2006-2007.

The present panel consists of the following members: A. James Barkovich, MD, Charlotte Bell, MD, (American Society of Anesthesiologists), James P. Borgstede, MD, FACR, William G. Bradley, MD, PhD, FACR, Jerry W. Froelich, MD, FACR, J. Rod Gimbel, MD, FACC, Cardiologist, John Gosbee, MD, MS, Ellisa Kuhni-Kaminski, RT (R)(MR), Emanuel Kanal, MD, FACR, FISMRM (chair), James W. Lester Jr., MD, John Nyenhuis, PhD, Daniel Joe Schaefer, PhD Engineer, Elizabeth A. Sebek, RN, BSN, CRN, Jeffrey Weinreb, MD, Terry Woods, PhD, FDA, Pamela Wilcox, RN, MBA (ACR Staff), Leonard Lucey, JD, LLM (ACR Staff), and Dina Hernandez, RT (R) (CT) (QM) (ACR Staff). The following represents the most recently 
modified and updated version of the combined prior three reports $(3,4,9)$ issued by the American College of Radiology Blue Ribbon Panel on MR Safety, chaired by Emanuel Kanal, MD, FACR. It is important to note that nothing that appears herein is the result of a "majority vote" of the member of this panel. As with each prior publication of these ACR MR Safe Practice Guidelines, the entire document, from introduction to the markedly expanded appendices, represents the unanimous consensus of each and every member of this Safety Committee and the various areas of expertise that they represent. This includes representation from fields and backgrounds as diverse as MR physicists, research/ academic radiologists, private practice radiologists, MR safety experts, patient safety experts/researchers, MR technologists, MR nursing, National Electrical Manufacturers Association, the Food and Drug Administration, the American Society of Anesthesiologists, legal counsel, and others. Lay personnel, physicians, Ph.D.s, department chairs and house-staff/residents, government employees and private practitioners, doctors, nurses, technologists, radiologists, anesthesiologists, cardiologists, attorneys-these are all represented on this Committee. It was believed that achieving unanimity for these Guidelines was critical to demonstrate to all that these Guidelines are not only appropriate from a scientific point of view, but reasonably applicable in the real world in which we all must live, with all its patient care, financial, and throughput pressures and considerations. The views expressed in this study are solely those of the authors and in no way suggest a policy or position of any of the organizations represented by the authors.

The following MR safe practice guidelines document is intended to be used as a template for MR facilities to follow in the development of an MR safety program. These guidelines were developed to help guide MR practitioners regarding these issues and to provide a basis for them to develop and implement their own MR policies and practices. It is intended that these MR safe practice guidelines (and the policies and procedures to which they give rise) be reviewed and updated on a regular basis as the field of MR safety continues to evolve.

The principles behind these MR Safe Practice Guidelines are specifically intended to apply not only to diagnostic settings but also to patient, research subject, and health care personnel safety for all MRI settings, including those designed for clinical diagnostic imaging, research, interventional, and intraoperative MR applications.

With the increasing advent and use of 3.0-Tesla and higher strength magnets, users need to recognize that one should never assume MR compatibility or safety information about a device if it is not clearly documented in writing. Decisions based on published MR safety and compatibility claims should recognize that all such claims apply only to specifically tested conditions, such as static magnetic field strengths, static gradient magnetic field strengths and spatial distributions, and the strengths and rates of change of gradient and radiofrequency (RF) magnetic fields.

Finally, there are many issues that impact MR safety which should be considered during site planning for a given MR installation. We include in this manuscript, as separate appendices, sections that address such issues as well, including cryogen emergency vent locations and pathways, 5-Gauss line, siting considerations, patient access pathways, etc. Yet despite their appearance herein, these issues, and many others, should be reviewed with those experienced with MR site planning and familiar with the patient safety and patient flow considerations before committing construction to a specific site design. In this regard, enlisting the assistance of an architectural firm experienced in this area, and doing so early in the design stages of the planning process, may prove most valuable.

It remains the intent of the ACR that these MR Safe Practice Guidelines will prove helpful as the field of MRI continues to evolve and mature, providing MR services that are among the most powerful, yet safest, of all diagnostic procedures to be developed in the history of modern medicine.

\section{ACR GUIDANCE DOCUMENT ON MR SAFE PRACTICES: 2013}

\section{A. Establish, Implement, and Maintain Current MR Safety Policies and Procedures}

1. All clinical and research MR sites, irrespective of magnet format or field strength, including installations for diagnostic, research, interventional, and/or surgical applications, should maintain MR safety policies.

2. These policies and procedures should also be reviewed concurrently with the introduction of any significant changes in safety parameters of the MR environment of the site (e.g., adding faster or stronger gradient capabilities or higher RF duty cycle studies) and updated as needed. In this review process, national and international standards and recommendations should be taken into consideration before establishing local guidelines, policies, and procedures

3. Each site will name a MR medical director whose responsibilities will include ensuring that MR safe practice guidelines are established and maintained as current and appropriate for the site. It is the responsibility of the site's administration to ensure that the policies and procedures that result from these MR safe practice guidelines are implemented and adhered to at all times by all of the site's personnel.

4. Procedures should be in place to ensure that any and all adverse events, MR safety incidents, or "near incidents" that occur in the MR site are to be reported to the medical director in a timely manner (e.g., within 24 hours or 1 business day of their occurrence) and used in continuous quality improvement efforts. It should be stressed that the Food and Drug Administration states that it is incumbent upon the sites to also report adverse events and incidents to them by means of their Medwatch program. The ACR supports this requirement and believes that it is in the 


\section{MRI Functional Diagram}

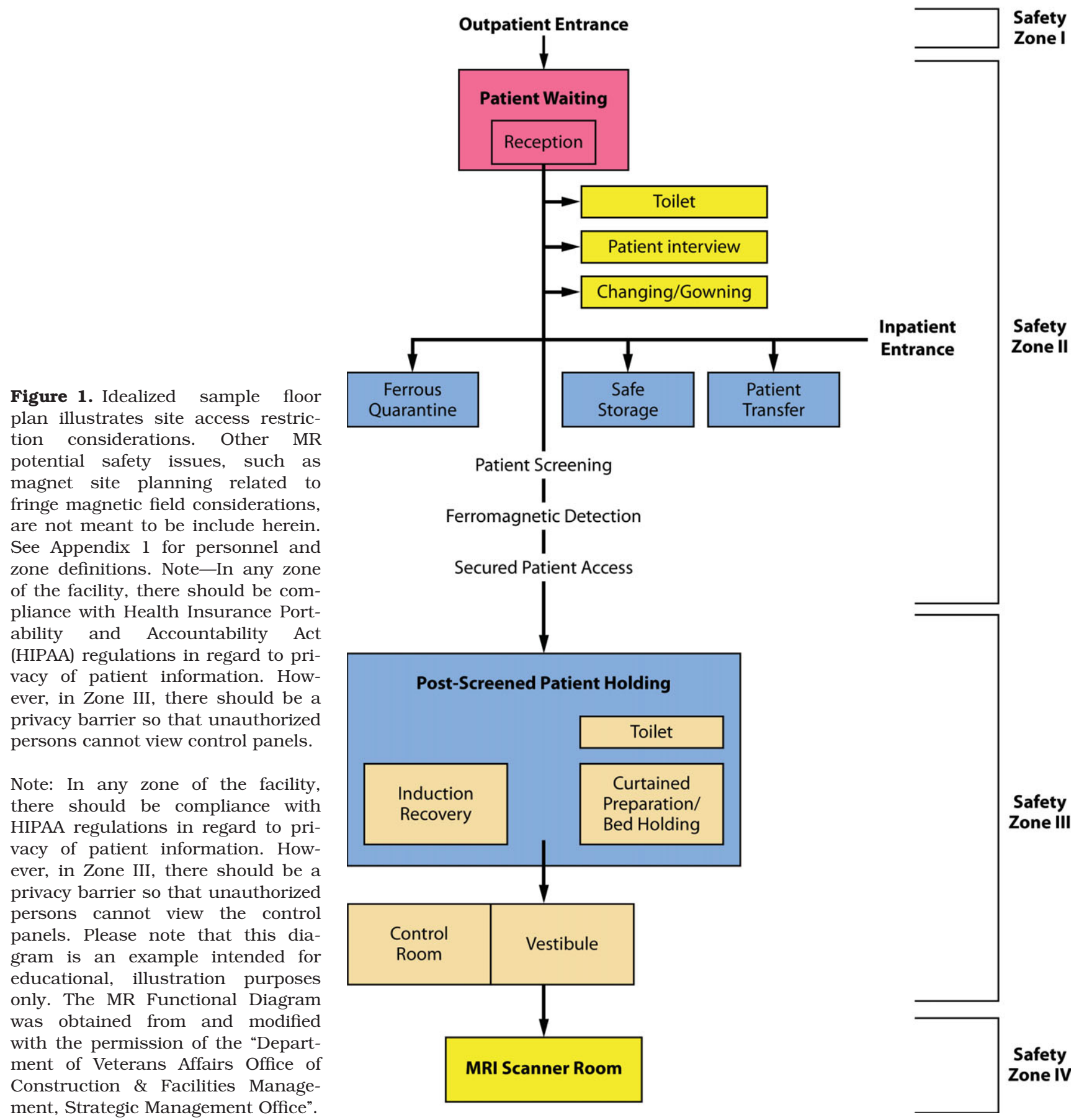

ultimate best interest of all MR practitioners to create and maintain this consolidated database of such events to help us all learn about them and how to better avoid them in the future (10).

\section{B. Static Magnetic Field Issues: Site Access Restriction}

\section{Zoning}

The MR site is conceptually divided into four Zones [see Fig. 1 and Appendices 1 and 3]: a. Zone I: This region includes all areas that are freely accessible to the general public. This area is typically outside the MR environment itself and is the area through which patients, health care personnel, and other employees of the MR site access the MR environment.

b. Zone II: This area is the interface between the publicly accessible, uncontrolled. Zone I and the strictly controlled Zones III and IV. Typically, patients are greeted in Zone II and are not free to move throughout Zone II at will, but are rather 
under the supervision of MR personnel (see section B.2.b, below). It is in Zone II that the answers to MR screening questions, patient histories, medical insurance questions, etc. are typically obtained.

c. Zone III: This area is the region in which free access by unscreened non-MR personnel or ferromagnetic objects or equipment can result in serious injury or death as a result of interactions between the individuals or equipment and the MR scanner's particular environment. These interactions include, but are not limited to, those involving the MR scanner's static and time-varying magnetic fields. All access to Zone III is to be strictly restricted, with access to regions within it (including Zone IV see below) controlled by, and entirely under the supervision of, MR personnel (see Section B.2.b, below). Specifically identified MR personnel (typically, but not necessarily only, the MR technologists) are to be charged with ensuring that this MR safe practice guideline is strictly adhered to for the safety of the patients and other non-MR personnel, the health care personnel, and the equipment itself. This function of the MR personnel is directly under the authority and responsibility of the MR medical director or the level 2-designated (see section B.2.b, below) physician of the day for the MR site.

Zone III regions should be physically restricted from general public access by, for example, key locks, passkey locking systems, or any other reliable, physically restricting method that can differentiate between MR personnel and non-MR personnel. The use of combination locks is discouraged as combinations often become more widely distributed than initially intended, resulting in site restriction violations being more likely with these devices. Only MR personnel shall be provided free access, such as the access keys or passkeys, to Zone III.

There should be no exceptions to this guideline. Specifically, this includes hospital or site administration, physician, security, and other non-MR personnel (see section B.2.c, below). Non-MR personnel are not to be provided with independent Zone III access until such time as they undergo the proper education and training to become MR personnel themselves. Zone III, or at the very least the area within it wherein the static magnetic field's strength exceeds 5-Gauss should be demarcated and clearly marked as being potentially hazardous. Because magnetic fields are three-dimensional volumes, Zone III controlled access areas may project through floors and ceilings of MRI suites, imposing magnetic field hazards on persons on floors other than that of the MR scanner. Zones of magnetic field hazard should be clearly delineated, even in typically nonoccupied areas such as rooftops or storage rooms, and access to these Zone III areas should be similarly restricted from non-MR personnel as they would be inside any other Zone III region associated with the MRI suite. For this reason, magnetic field strength plots for all MRI systems should be analyzed in vertical section as well as in horizontal plan, identifying areas above or below, in addition to areas on the same level, where persons may be at risk of interactions with the magnetic field.

d. Zone IV: This area is synonymous with the MR scanner magnet room itself, i.e., the physical confines of the room within which the MR scanner is located (see Appendix 3). Zone IV, by definition, will always be located within Zone III, as it is the MR magnet and its associated magnetic field that generates the existence of Zone III. Zone IV should also be demarcated and clearly marked as being potentially hazardous due to the presence of very strong magnetic fields. As part of the Zone IV site restriction, all MR installations should provide for direct visual observation by level 2 personnel to access pathways into Zone IV. By means of illustration only, the MR technologists would be able to directly observe and control, by means of line of site or by means of video monitors, the entrances or access corridors to Zone IV from their normal positions when stationed at their desks in the scan control room.

Zone IV should be clearly marked with a red light and lighted sign stating, "The Magnet is On". Ideally, signage should inform the public that the magnetic field is active even when power to the facility is deactivated. Except for resistive systems, this light and sign should be illuminated at all times and should be provided with a battery backup energy source to continue to remain illuminated in the event of a loss of power to the site.

In case of cardiac or respiratory arrest or other medical emergency within Zone IV for which emergent medical intervention or resuscitation is required, appropriately trained and certified MR personnel should immediately initiate basic life support or CPR as required by the situation while the patient is being emergently removed from Zone IV to a predetermined, magnetically safe location. All priorities should be focused on stabilizing (e.g., basic life support with cardiac compressions and manual ventilation) and then evacuating the patient as rapidly and safely as possible from the magnetic environment that might restrict safe resuscitative efforts.

Furthermore, for logistical safety reasons, the patient should always be moved from Zone IV to the prospectively identified location where full resuscitative efforts are to continue (see Appendix 3).

Quenching the magnet (for superconducting systems only) is not routinely advised for cardiac or respiratory arrest or other medical emergency, because quenching the magnet and having the magnetic field dissipate could easily take more than a minute. Furthermore, as quenching a magnet can theoretically be hazardous, ideally one should evacuate the magnet room, when possible, for an intentional quench. One should rather use that time wisely to initiate life support 
measures while removing the patient from Zone IV to a location where the strength of the magnetic field is insufficient to be a medical concern. Zones III and IV site access restriction must be maintained during resuscitation and other emergent situations for the protection of all involved.

\section{MR Personnel and non-MR personnel}

a. All individuals working within at least Zone III of the MR environment should be documented as having successfully completed at least one of the MR safety live lectures or prerecorded presentations approved by the MR medical director. Attendance should be repeated at least annually, and appropriate documentation should be provided to confirm these ongoing educational efforts. These individuals shall be referred to henceforth as MR personnel.

b. There are two levels of MR personnel:

1. Level 1 MR personnel: Those who have passed minimal safety educational efforts to ensure their own safety as they work within Zone III will be referred to henceforth as level $1 \mathrm{MR}$ personnel.

2. Level 2 MR personnel: Those who have been more extensively trained and educated in the broader aspects of MR safety issues, including, for example, issues related to the potential for thermal loading or burns and direct neuromuscular excitation from rapidly changing gradients, will be referred to henceforth as level $2 \mathrm{MR}$ personnel. It is the responsibility of the MR medical director not only to identify the necessary training, but also to identify those individuals who qualify as level $2 \mathrm{MR}$ personnel. It is understood that the medical director will have the necessary education and experience in MR safety to qualify as level 2 MR personnel. (See Appendix 1.)

c. All those not having successfully complied with these MR safety instruction guidelines shall be referred to henceforth as non-MR personnel. Specifically, non-MR personnel will be the terminology used to refer to any individual or group who has not within the previous 12 months undergone the designated formal training in MR safety issues defined by the MR safety director of that installation.

\section{Patient and non-MR personnel screening}

a. All non-MR personnel wishing to enter Zone III must first pass an MR safety screening process. Only MR personnel are authorized to perform an MR safety screen before permitting non-MR personnel into Zone III.

b. The screening process and screening forms for patients, non-MR personnel, and MR personnel should be essentially identical. Specifically, one should assume that screened non-MR personnel, health care practitioners, or MR personnel may enter the bore of the MR imager during the MR imaging process.

Examples of this might include if a pediatric patient cries for his mother, who then leans into the bore, or if the anesthetist leans into the bore to manually ventilate a patient in the event of a problem.

c. Metal detectors

The usage in MR environments of conventional metal detectors which do not differentiate between ferrous and nonferromagnetic materials is not recommended. Reasons for this recommendation against conventional metal detector usage include, among others:

1. They have varied-and variable-sensitivity settings.

2. The skills of the operators can vary.

3. Today's conventional metal detectors cannot detect, for example, a $2 \times 3 \mathrm{~mm}$, potentially dangerous ferromagnetic metal fragment in the orbit or near the spinal cord or heart.

4. Today's conventional metal detectors do not differentiate between ferromagnetic and nonferromagnetic metallic objects, implants, or foreign bodies.

5. Metal detectors should not be necessary for the detection of large metallic objects, such as oxygen tanks on the gurney with the patients. These objects are fully expected to be detected and physically excluded - during the routine patient screening process.

However, ferromagnetic detection systems are currently available that are simple to operate, capable of detecting even very small ferromagnetic objects external to the patient, and differentiating between ferromagnetic and non-ferromagnetic materials. While the use of conventional metal detectors is not recommended, the use of ferromagnetic detection systems is recommended as an adjunct to thorough and conscientious screening of persons and devices approaching Zone IV. It should be reiterated that their use is in no way meant to replace a thorough screening practice, which rather should be supplemented by their usage.

d. Non-MR personnel should be accompanied by, or under the immediate supervision of and in visual or verbal contact with, one specifically identified level 2 MR person for the entirety of their duration within Zone III or IV restricted regions. However, it is acceptable to have them in a changing room or restroom in Zone III without visual contact as long as the personnel and the patient can communicate verbally with each other.

Level 1 MR personnel are permitted unaccompanied access throughout Zones III and IV. Level 1 MR personnel are also explicitly permitted to be responsible for accompanying non-MR personnel into and throughout Zone III, excluding Zone IV. However, level $1 \mathrm{MR}$ personnel are not permitted to directly admit, or be designated responsible for, non-MR personnel in Zone IV.

In the event of a shift change, lunch break, etc., no level 2 MR personnel shall relinquish their responsibility to supervise non-MR personnel still within Zone III or IV until such supervision has been formally transferred to another of the site's level 2 MR personnel. 
e. Nonemergent patients should be MR safety screened on site by a minimum of 2 separate individuals. At least one of these individuals should be level $2 \mathrm{MR}$ personnel. At least one of these 2 screens should be performed verbally or interactively.

Emergent patients and their accompanying nonMR personnel may be screened only once, providing the screening individual is level 2 MR personnel. There should be no exceptions to this.

f. Any individual undergoing an MR procedure must remove all readily removable metallic personal belongings and devices on or in them (e.g., watches, jewelry, pagers, cell phones, body piercings (if removable), contraceptive diaphragms, metallic drug delivery patches (see Section I, below), cosmetics containing metallic particles (such as eye make-up), and clothing items which may contain metallic fasteners, hooks, zippers, loose metallic components or metallic threads). It is therefore advisable to require that the patients or research subjects wear a site-supplied gown with no metal fasteners when feasible.

g. All patients and non-MR personnel with a history of potential ferromagnetic foreign object penetration must undergo further investigation before being permitted entrance to Zone III. Examples of acceptable methods of screening include patient history, plain X-ray films, prior CT or MR studies of the questioned anatomic area, or access to written documentation as to the type of implant or foreign object that might be present. Once positive identification has been made as to the type of implant or foreign object that is within a patient, best effort assessments should be made to identify the MR compatibility or MR safety of the implant or object. Efforts at identification might include written records of the results of formal testing of the implant before implantation (preferred), product labeling regarding the implant or object, and peer-reviewed publications regarding MR compatibility and MR safety testing of the specific make, model, and type of the object. MR safety testing would be of value only if the object or device had not been altered since such testing had been published and only if it can be confirmed that the testing was performed on an object of precisely the same make, model, and type.

All patients who have a history of orbit trauma by a potential ferromagnetic foreign body for which they sought medical attention are to have their orbits cleared by either plain X-ray orbit films (2 views) $(11,12)$ or by a radiologist's review and assessment of contiguous cut prior CT or MR images (obtained since the suspected traumatic event) if available.

h. Conscious, nonemergent patients and research and volunteer subjects are to complete written MR safety screening questionnaires before their introduction to Zone III. Family or guardians of nonresponsive patients or of patients who cannot reliably provide their own medical histories are to complete a written MR safety screening questionnaire before their introduction to Zone III. These completed questionnaires are then to be reviewed orally with the patient, guardian, or research subject in their entirety before permitting the patient or research subject to be cleared into Zone III.

The patient, guardian, or research subject as well as the screening MR staff member must both sign the completed form. This form should then become part of the patient's medical record. No empty responses will be accepted-each question must be answered with a "yes" or "no" or specific further information must be provided as requested. A sample pre-MR screening form is provided (see Appendix 2). This is the minimum information to be obtained; more may be added if the site so desires.

i. Screening of the patient or non-MR personnel with, or suspected of having, an intracranial aneurysm clip should be performed as per the separate MR safe practice guideline addressing this particular topic (see section M, below).

j. Screening of patients for whom an MR examination is deemed clinically indicated or necessary, but who are unconscious or unresponsive, who cannot provide their own reliable histories regarding prior possible exposures to surgery, trauma, or metallic foreign objects, and for whom such histories cannot be reliably obtained from others:

1. If no reliable patient metal exposure history can be obtained, and if the requested MR examination cannot reasonably wait until a reliable history might be obtained, it is recommended that such patients be physically examined by level 2 MR personnel. All areas of scars or deformities that might be anatomically indicative of an implant, such as on the chest or spine region, and whose origins are unknown and which may have been caused by ferromagnetic foreign bodies, implants, etc., should be subject to plain-film radiography (if recently obtained plain films or CT or MR studies of such areas are not already available). The investigation described above should be made to ensure there are no potentially harmful embedded or implanted metallic foreign objects or devices. All such patients should also undergo plain film imaging of the skull or orbits and chest to exclude metallic foreign objects (if recently obtained such radiographic or MR information not already available).

2. Monitoring of patients in the MR scanner is sometimes necessary. However, monitoring methods should be chosen carefully due to the risk of thermal injury associated with monitoring equipment in the MR environment. Sedated, anesthetized, or unconscious patients may not be able to express symptoms of such injury. This potential for injury is greater on especially higher field whole body scanners (e.g., 1 Tesla and above), but exists at least theoretically at all MR imaging field strengths. MR Conditional EKG electrodes should be used and leads should be kept from touching the patients during the scan. Patients who require EKG 
monitoring and who are unconscious, sedated, or anesthetized should be examined after each imaging sequence with potential repositioning of the EKG leads and any other electrically conductive material with which the patient is in contact. Alternatively, cold compresses or ice packs could be placed upon all necessary electrically conductive material that touches the patient during scanning.

Distortion of the electrocardiogram within the magnetic field can make interpretation of the ECG complex unreliable, even with filtering used by contemporary monitoring systems. Routine monitoring of heart rate and rhythm may also be accomplished using pulse oximetry, which would eliminate the risks of thermal injury from electrocardiography.

k. Final determination of whether or not to scan any given patient with any given implant, foreign body, etc. is to be made by the level 2 designated attending MR radiologist, the MR medical director, or specifically designated level 2 MR personnel following criteria for acceptability predetermined by the medical director. These risks include, among others, consideration of mechanical and thermal risks associated with MR imaging of implants, as well as assessments of the safety of exposure of the device to the electromagnetic forces used in the MR imaging process.

For implants that are strongly ferromagnetic, an obvious concern is that of magnetic translational and rotational forces upon the implant which might move or dislodge the device from its implanted position If an implant has demonstrated weak ferromagnetic forces on formal testing, it might be prudent to wait several weeks for fibrous scarring to set in, as this may help anchor the implant in position and help it resist such weakly attractive magnetic forces that might arise in MR environments.

For all implants that have been demonstrated to be nonferrous in nature, however, the risk of implant motion is essentially reduced to those resulting from Lenz's forces alone. These tend to be quite trivial for typical metallic implant sizes of a few centimeters or less. Thus, a waiting period for fibrous scarring to set in is far less important, and the advisability for such a waiting period may well be easily outweighed by the potential clinical benefits of undergoing an MR examination at that time. As always, clinical assessment of the risk benefit ratio for the particular clinical situation and patient at hand are paramount for appropriate medical decision making in these scenarios.

It is possible that during the course of a magnetic resonance imaging examination an unanticipated ferromagnetic implant or foreign body is discovered within a patient or research subject undergoing the examination. This is typically suspected or detected by means of a sizable field-distorting artifact seen on spin echo imaging techniques that grows more obvious on longer TE studies and expands markedly on typical moderate or long TE gradient echo imaging sequences. In such cases, it is imperative that the medical director, safety officer, and/or physician in charge be immediately notified of the suspected findings. This individual should then assess the situation, review the imaging information obtained, and decide what the best course of action might be.

It should be noted that there are numerous potentially acceptable courses that might be recommended which in turn are dependent upon many factors, including the status of the patient, the location of the suspected ferromagnetic implant/foreign body relative to local anatomic structures, the mass of the implant, etc. Appropriate course of actions might include proceeding with the scan under way, immobilizing the patient and the immediate removal from the scanner, or other intermediate steps. Regardless of the course of action selected, it is important to note that the forces on the implant will change, and may actually increase, during the attempt to remove the patient from the scanner bore. Furthermore, the greater the rate of motion of the patient/device through the magnetic fields of the scanner bore the greater the forces acting upon that device will likely be. Thus it is prudent to ensure that if at all possible, immobilization of the device during patient extraction from the bore, and slow, cautious, deliberate rate of extricating the patient from the bore, will likely result in weaker and potentially less harmful forces on the device as it traverses the various static magnetic field gradients associated with the MR imager.

It is also worthy of note that the magnetic fields associated with the MR scanner are three dimensional. Thus, especially for superconducting systems, one should avoid the temptation to have the patient sit up as soon as they are physically out of the bore. Doing so may expose the ferrous object to still significant torque- and translation-related forces despite their being physically outside the scanner bore. It is therefore advisable to continue to extract the patient along a straight line course parallel to the center of the magnet while the patient remains immobilized until they are as far as physically possible from the MR imager itself, before any other patient/object motion vector is attempted or permitted.

1. All non-MR personnel (e.g., patients, volunteers, varied site employees and professionals) with implanted cardiac pacemakers, implantable cardioverter defibrillators (ICDs), diaphragmatic pacemakers, electromechanically activated devices, or other electrically conductive devices upon which the non-MR personnel is dependent should be precluded from Zone IV and physically restrained from the 5-Gauss line unless specifically cleared in writing by a level 2 designated attending radiologist or the medical director of the MR site. In such circumstances, specific defending risk-benefit rationale should be provided in writing and signed by the authorizing radiologist.

Should it be determined that non-MR personnel wishing to accompany a patient into an MR scan 
room require their orbits to be cleared by plain-film radiography, a radiologist must first discuss with the non-MR personnel that plain X-ray films of their orbits are required before permitting them access to the MR scan room. Should they still wish to proceed with access to Zone IV or within the 5-G line, and should the attending radiologist deem it medically advisable that they do so (e.g., for the care of their child about to undergo an MR study), written informed consent should be provided by these accompanying non-MR personnel before their undergoing X-ray examination of their orbits.

m. MR scanning of patients, prisoners, or parolees with metallic prisoner-restraining devices or RF ID or tracking bracelets could lead to theoretical adverse events, including: (i) ferromagnetic attractive effects and resultant patient injury, (ii) possible ferromagnetic attractive effects and potential damage to the device or its battery pack, (iii) RF interference with the MRI study and secondary image artifact, (iv) RF interference with the functionality of the device, (v) RF power deposition and heating of the bracelet or tagging device or its circuitry and secondary patient injury (if the bracelet would be in the anatomic volume of the RF transmitter coil being used for imaging). Therefore, in cases where requested to scan a patient, prisoner, or parolee wearing RF bracelets or metallic handcuffs or anklecuffs, request that the patient be accompanied by the appropriate authorities who can and will remove the restraining device before the MR study and be charged with its replacement following the examination.

n. Firefighter, police, and security safety considerations: For the safety of firefighters and other emergent services responding to an emergent call at the MR site, it is recommended that all fire alarms, cardiac arrests, or other emergent service response calls originating from or located in the MR site should be forwarded simultaneously to a specifically designated individual from amongst the site's MR personnel. This individual should, if possible, be on site before the arrival of the firefighters or emergent responders to ensure that they do not have free access to Zone III or IV. The site might consider assigning appropriately trained security personnel, who have been trained and designated as MR personnel, to respond to such calls.

In any case, all MR sites should arrange to prospectively educate their local fire marshals, firefighters associations, and police or security personnel about the potential hazards of responding to emergencies in the MR suite.

It should be stressed that even in the presence of a true fire (or other emergency) in Zone III or IV, the magnetic fields may be present and fully operational. Therefore, free access to Zone III or IV by firefighters or other non-MR personnel with air tanks, axes, crowbars, other firefighting equipment, guns, etc might prove catastrophic or even lethal to those responding or others in the vicinity.

As part of the Zone III and IV restrictions, all MR sites must have clearly marked, readily accessible
MR Conditional or MR Safe fire extinguishing equipment physically stored within Zone III or IV.

All conventional fire extinguishers and other firefighting equipment not tested and verified safe in the MR environment should be restricted from Zone III.

For superconducting magnets, the helium (and the nitrogen as well, in older MR magnets) is not flammable and does not pose a fire hazard directly. However, the liquid oxygen that can result from the supercooled air in the vicinity of the released gases might well increase the fire hazard in this area. If there are appropriately trained and knowledgeable MR personnel available during an emergency to ensure that emergency response personnel are kept out of the MR scanner or magnet room and 5-Gauss line, quenching the magnet during a response to an emergency or fire should not be a requirement.

However, if the fire is in such a location where Zone III or IV needs to be entered for whatever reason by firefighting or emergency response personnel and their firefighting and emergent equipment, such as air tanks, crowbars, axes, defibrillators, a decision to quench a superconducting magnet should be very seriously considered to protect the health and lives of the emergent responding personnel. Should a quench be performed, appropriately designated MR personnel still need to ensure that all non-MR personnel (including and especially emergently response personnel) continue to be restricted from Zones III and IV until the designated MR personnel has personally verified that the static field is either no longer detectable or at least sufficiently attenuated as to no longer present a potential hazard to one moving by it with, for example, large ferromagnetic objects such as air tanks or axes.

For resistive systems, the magnetic field of the MR scanner should be shut down as completely as possible and verified as such before permitting the emergency response personnel access to Zone IV. For permanent, resistive, or hybrid systems whose magnetic fields cannot be completely shut down, MR personnel should ideally be available to warn the emergency response personnel that a very powerful magnetic field is still operational in the magnet room.

\section{MR Personnel Screening}

All MR personnel are to undergo an MR-screening process as part of their employment interview process to ensure their safety in the MR environment. For their own protection and for the protection of the nonMR personnel under their supervision, all MR personnel must immediately report to the MR medical director any trauma, procedure, or surgery they experience or undergo where a ferromagnetic object or device may have become introduced within or on them. This will permit appropriate screening to be performed on the employee to determine the safety of permitting that employee into Zone III. 


\section{Device and Object Screening}

Ferrous objects, including those brought by patients, visitors, contractors, etc., should be restricted from entering Zone III, whenever practical.

As part of the Zone III site restriction and equipment testing and clearing responsibilities, all sites should have ready access to a strong handheld magnet ( $\geq 1000$-Gauss) and/or a handheld ferromagnetic detection device. This will enable the site to test external, and even some superficial internal devices or implants for the presence of grossly detectable ferromagnetic attractive forces.

a. All portable metallic or partially metallic devices that are on or external to the patient (e.g., oxygen cylinders) are to be positively identified in writing as MR Unsafe or, alternatively, MR Safe or MR Conditional in the MR environment before permitting them into Zone III Figure 2. For all device or object screening, verification and positive identification should be in writing. Examples of devices that need to be positively identified include fire extinguishers, oxygen tanks and aneurysm clips.

b. External devices or objects demonstrated to be ferromagnetic and MR Unsafe or incompatible in the MR environment may still, under specific circumstances, be brought into Zone III if for example, they are deemed by MR personnel to be necessary and appropriate for patient care. They should only be brought into Zone III if they are under the direct supervision of specifically designated level 1 or level $2 \mathrm{MR}$ personnel who are thoroughly familiar with the device, its function, and the reason supporting its introduction to Zone III. The safe usage of these devices while they are present in Zone III will be the responsibility of specifically named level 1 or 2 MR personnel. These devices must be appropriately physically secured or restricted at all times during which they are in Zone III to ensure that they do not inadvertently come too close to the MR scanner and accidentally become exposed to static magnetic fields or gradients that might result in their becoming either hazardous projectiles or no longer accurately functional.

c. Never assume MR compatibility or safety information about the device if it is not clearly documented in writing. All unknown external objects or devices being considered for introduction beyond Zone II should be tested with a strong handheld magnet ( $\geq$ 1000-Gauss) and/or a handheld ferromagnetic detection device for ferromagnetic properties before permitting them entry to Zone III. The results of such testing, as well as the date, time, and name of the tester, and methodology used for that particular device, should be documented in writing. If a device has not been tested, or if its MR compatibility or safety status is unknown, it should not be permitted unrestricted access to Zone III.

d. All portable metallic or partially metallic objects that are to be brought into Zone IV must be prop-

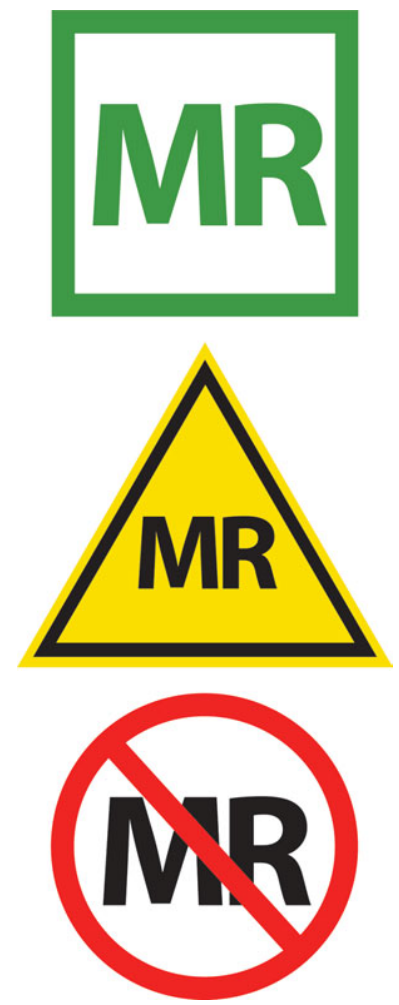

Figure 2. U.S. Food and Drug Administration labeling criteria (developed by ASTM [American Society for Testing and Materials] International) for portable objects taken into Zone IV. Square green "MR safe" label is for wholly nonmetallic objects, triangular yellow label is for objects with "MR conditional" rating, and round red label is for "MR unsafe" objects.

erly identified and appropriately labeled using the current FDA labeling criteria developed by ASTM International in standard ASTM F2503 (http://www.astm.org). Those items which are wholly, nonmetallic should be identified with a square green "MR Safe" label. Items which are clearly ferromagnetic should be identified as "MR Unsafe" and labeled appropriately with the corresponding round red label. Objects with an MR Conditional rating should be affixed with a triangular yellow MR Conditional label before being brought into the scan room/Zone IV.

As noted in the introduction to this section B.5, above, if MR safety data is not prospectively available for a piece of equipment or object that requires electricity (or battery power) to operate, it should not be brought into Zone IV without being subjected to the testing outlined in ASTM F2503. If MR safety data is not prospectively available for a given object that is not electrically activated (e.g., wash basins, scrub brushes, step stools), initial testing for the purpose of this labeling is to be accomplished by the site's MR personnel exposing the object to a handheld magnet $(\geq 1000$-Gauss). If grossly detectable attractive forces are observed between the object being tested or any of its components and the handheld magnet, it is to be labeled with a 
circular red "MR Unsafe" label. If no attractive forces are observed, a triangular yellow "MR Conditional" label is to be attached to the object. It is only when the composition of an object and its components are known to be nonmetallic and not electrically conductive that the green "MR Safe" label is to be affixed to a device or object. Particularly with regard to nonclinical and incidental equipment, current products marketed with ill-defined terminology such as "nonmagnetic", or outdated classifications such as "MR compatible", should not be presumed to conform to a particular current ASTM classification. Similarly, any product marketed as "MR safe" but with metallic construction or components should be treated with suspicion. Objects intended for use in Zone IV, including nonclinical incidental products such as stepping stools or ladders, which are not accompanied by manufacturer or third-party MR safety test results under the ASTM F2503 criteria, should be site tested as described above.

e. Decisions based on published MR compatibility or safety claims should recognize that all such claims apply to specifically tested static field and static gradient field, strengths and only to the precise model, make, and identification of the tested object. For example, "MR Conditional having been tested to be safe at 3.0 Tesla at gradient strengths of $400-\mathrm{G} / \mathrm{cm}$ or less and normal operating mode.",

f. It should be noted that alterations performed by the site on MR Safe, MR Unsafe, and MR Conditional equipment or devices may alter the MR safety or compatibility properties of the device. For example, tying a ferromagnetic metallic twisting binder onto a sign labeling the device as MR Conditional or MR Safe might result in artifact induction - or worse - if introduced into the MR scanner.

\section{Lenz's Forces:}

Faraday's Law states that a moving or changing magnetic field will induce a voltage in a perpendicularly oriented electrical conductor. Lenz's Law builds upon this and states that the induced voltage will itself be such that it will secondarily generate its own magnetic field whose orientation and magnitude will oppose that of the initial time varying magnetic field that created it in the first place. For example, if an electrical conductor is moved perpendicularly toward the magnetic field $\mathrm{BO}$ of an MR scanner, even if this conductor is not grossly ferromagnetic, the motion itself will result in the generation of voltages within this conductor whose magnitude is directly proportional to the rate of motion as well as the spatial gradient of magnetic field BO through which it is being moved. Conducting objects turning in the static field will also experience a torque due to the induced eddy currents. Lenz's law states that this induced current will in turn create a magnetic field whose orientation will oppose the BO magnetic field that created this current.

Thus, moving a large metallic but nonferromagnetic electrical conductor toward the magnet bore will result in the induction of a voltage and associated magnetic field which will orient in such a manner and at such a strength to oppose the motion of the metallic object into the bore of the MR scanner. If, for example, one tries to move a nonferrous oxygen tank into the bore of an MR scanner, as the scanner bore is approached Lenz's forces will be sufficiently strong to virtually stop forward progress of the tank. Furthermore, the faster one moves the tank into the bore, the greater the opposing force that is created to stop this motion.

This also has potential consequence for large implanted metallic devices such as certain metallic nonferrous infusion pumps. Although they may not pose a projectile hazard, rapid motion of the patient/ implant perpendicular to the magnetic field of the MR imager can be expected to result in forces on the implant that would oppose this motion and may likely be detected by the patient. If the patient were to complain of experiencing forces tugging or pulling on the implant, this might bring the patient or health care personnel to erroneously conclude that there were ferrous components to the device, and possible cancellation of the examination. Slowly moving such large metallic devices into and out of the bore is a key factor in decreasing any Lenz's forces that might be induced, and decrease the likelihood of a misunderstanding or unnecessary study cancellation.

\section{MR Technologist}

1. MR technologists should be in compliance with the technologist qualifications listed in the MR Accreditation Program Requirements.

2. Except for emergent coverage, there will be a minimum of 2 MR technologists or one MR technologist and one other individual with the designation of MR personnel in the immediate Zone II through Zone IV MR environment. For emergent coverage, the MR technologist can scan with no other individuals in their Zone II through Zone IV environment as long as there is in-house, ready emergent coverage by designated department of radiology MR personnel (e.g., radiology house staff or radiology attending).

\section{Pregnancy Related Issues}

\section{Health Care Practitioner Pregnancies:}

Pregnant health care practitioners are permitted to work in and around the MR environment throughout all stages of their pregnancy (13). Acceptable activities include, but are not limited to, positioning patients, scanning, archiving, injecting contrast, and entering the MR scan room in response to an emergency. Although permitted to work in and around the MR environment, pregnant health care practitioners are 
requested not to remain within the MR scanner bore or Zone IV during actual data acquisition or scanning.

\section{Patient Pregnancies}

Present data have not conclusively documented any deleterious effects of MR imaging exposure on the developing fetus. Therefore no special consideration is recommended for the first, versus any other, trimester in pregnancy. Nevertheless, as with all interventions during pregnancy, it is prudent to screen females of reproductive age for pregnancy before permitting them access to MR imaging environments. If pregnancy is established consideration should be given to reassessing the potential risks versus benefits of the pending study in determining whether the requested MR examination could safely wait to the end of the pregnancy before being performed.

a. Pregnant patients can be accepted to undergo MR scans at any stage of pregnancy if, in the determination of a level 2 MR personnel-designated attending radiologist, the risk-benefit ratio to the patient warrants that the study be performed. The radiologist should confer with the referring physician and document the following in the radiology report or the patient's medical record:

1. The information requested from the MR study cannot be acquired by means of nonionizing means (e.g., ultrasonography).

2. The data is needed to potentially affect the care of the patient or fetus during the pregnancy.

3. The referring physician believes that it is not prudent to wait until the patient is no longer pregnant to obtain this data.

b. MR contrast agents should not be routinely provided to pregnant patients. This decision too, is on that must be made on a case-by-case basis by the covering level $2 \mathrm{MR}$ personnel-designated attending radiologist who will assess the risk-benefit ratio for that particular patient.

The decision to administer a gadolinium-based MR contrast agent to pregnant patients should be accompanied by a well-documented and thoughtful risk-benefit analysis. This analysis should be able to defend a decision to administer the contrast agent based on overwhelming potential benefit to the patient or fetus outweighing the theoretical but potentially real risks of long-term exposure of the developing fetus to free gadolinium ions.

Studies have demonstrated that at least some of the gadolinium-based MR contrast agents readily pass through the placental barrier and enter the fetal circulation. From here, they are filtered in the fetal kidneys and then excreted into the amniotic fluid. In this location the gadolinium-chelate molecules are in a relatively protected space and may remain in this amniotic fluid for an indeterminate amount of time before finally being reabsorbed and eliminated. As with any equilibrium situation involving any dissociation constant, the longer the chelated molecule remains in this space, the greater the potential for dissociation of the poten- tially toxic gadolinium ion from its ligand. It is unclear what impact such free gadolinium ions might have if they were to be released in any quantity in the amniotic fluid. Certainly, deposition into the developing fetus would raise concerns of possible secondary adverse effects.

The risk to the fetus of gadolinium based MR contrast agent administration remains unknown and may be harmful.

\section{E. Pediatric MR Safety Concerns}

\section{Sedation and Monitoring Issues}

Children form the largest group requiring sedation for MRI, largely because of their inability to remain motionless during scans. Sedation protocols may vary from institution to institution according to procedures performed (diagnostic vs. interventional), the complexity of the patient population (healthy preschoolers vs. premature infants), the method of sedation (mild sedation vs. general anesthesia) and the qualifications of the sedation provider.

Adherence to standards of care mandates following the sedation guidelines developed by the American Academy of Pediatrics $(14,15)$, the American Society of Anesthesiologists (16), and the Joint Commission on Accreditation of Healthcare Organizations (17). In addition, sedation providers must comply with protocols established by the individual state and the practicing institution. These guidelines require the following provisions:

a. Preprocedural medical history and examination for each patient

b. Fasting guidelines appropriate for age

c. Uniform training and credentialing for sedation providers

d. Intraprocedural and post procedural monitors with adaptors appropriately sized for children (MR Conditional equipment)

e. Method of patient observation (window, camera)

f. Resuscitation equipment, including oxygen delivery and suction

g. Uniform system of record keeping and charting (with continuous assessment and recording of vital signs)

h. Location and protocol for recovery and discharge

i. Quality assurance program that tracks complications and morbidity.

For the neonatal and the young pediatric population, special attention is needed in monitoring body temperature for both hypo- and hyperthermia in addition to other vital signs (18). Temperature monitoring equipment that is approved for use in the MR suite is readily available. Commercially available, MR-approved neonatal isolation transport units and other warming devices are also available for use during MR scans.

\section{Pediatric Screening Issues}

Children may not be reliable historians and, especially for older children and teenagers, should be 
questioned both in the presence of parents or guardians and separately to maximize the possibility that all potential dangers are disclosed. Therefore, it is recommended that they be gowned before entering Zone IV to help ensure that no metallic objects, toys, etc. inadvertently find their way into Zone IV. Pillows, stuffed animals, and other comfort items brought from home represent real risks and should be discouraged from entering Zone IV. If unavoidable, each should be carefully checked with a powerful handheld magnet and/ or ferromagnetic detector and perhaps again in the MR scanner before permitting the patient to enter Zone IV with them to ensure that they do not contain any objectionable metallic components.

\section{MR Safety of Accompanying Family or Personnel:}

Although any age patient might request that others accompany them for their MR examination, this is far more common in the pediatric population. Those accompanying or remaining with the patient should be screened using the same criteria as anyone else entering Zone IV.

In general, it would be prudent to limit accompanying adults to a single individual. Only a qualified, responsible MR physician should make screening criteria exceptions.

Hearing protection and MR safe/MR conditional seating are recommended for accompanying family members within the MR scan room.

\section{F. Time Varying Gradient Magnetic Field Related Issues: Induced Voltages}

Types of patients needing extra caution:

Patients with implanted or retained wires in anatomically or functionally sensitive areas (e.g., myocardium or epicardium, implanted electrodes in the brain) should be considered at higher risk, especially from faster MRI sequences, such as echo planar imaging (which may be used in such sequences as diffusionweighted imaging, functional imaging, perfusion weighted imaging, MR angiographic imaging, etc.). The decision to limit the $\mathrm{dB} / \mathrm{dt}$ (rate of magnetic field change) and maximum strength of the magnetic field of the gradient subsystems during imaging of such patients should be reviewed by the level 2 MR personnel-designated attending radiologist supervising the case or patient.

\section{G. Time Varying Gradient Magnetic Field Related Issues: Auditory Considerations}

1. All patients and volunteers should be offered and encouraged to use hearing protection before undergoing any imaging in any MR scanners. FDA's current MR Guidance Document (Attachment B entitled, "Recommended User Instructions for a Magnetic Resonance Diagnostic Device") states that instructions from manufacturers of MR equipment should state that hearing protection is required for all patients studied on MR imaging systems capable of producing sound pressures that exceed $99 \mathrm{~dB}(\mathrm{~A})$. The International standard on this issue (IEC 60601-2-33: "Particular requirements for the basic safety and essential performance of magnetic resonance equipment for medical diagnosis"), also states that, for all equipment capable of producing more than an A-weighted rms sound pressure level of $99 \mathrm{~dB}(\mathrm{~A})$, hearing protection shall be used for the safety of the patient and that this hearing protection shall be sufficient to reduce the Aweighted r.m.s. sound pressure level to below 99 $\mathrm{dB}(\mathrm{A})$.

2. All patients or volunteers in whom research sequences are to be performed (i.e., MR scan sequences that have not yet been approved by the Food and Drug Administration) are to have hearing protective devices in place before initiating any MR sequences. Without hearing protection in place, MRI sequences that are not FDA approved should not be performed on patients or volunteers.

\section{H. Time Varying Radiofrequency Magnetic Field Related Issues: Thermal}

1. All unnecessary or unused electrically conductive materials external to the patient should be removed from the MR system before the onset of imaging. It is not sufficient to merely to "unplug" or disconnect unused, unnecessary electrically conductive material and leave it within the MR scanner with the patient during imaging. All electrical connections, such as on surface coil leads or monitoring devices must be visually checked by the scanning MR technologist before each usage to ensure the integrity of the thermal and electrical insulation.

2. Electrical voltages and currents can be induced within electrically conductive materials that are within the bore of the MR imager during the MR imaging process. This might result in the heating of this material by resistive losses. This heat might be of a caliber sufficient to cause injury to human tissue. As noted in section H.9, among the variables that determine the amount of induced voltage or current is the consideration that the larger the diameter of the conductive loops the greater the potential induced voltages or currents and, thus the greater the potential for resultant thermal injury to adjacent or contiguous patient tissue.

Therefore, when electrically conductive material (wires, leads, implants, etc.), are required to remain within the bore of the MR scanner with the patient during imaging, care should be taken to ensure that no large-caliber electrically conducting loops (including patient tissue; see section H. 5, below) are formed within the MR scanner during imaging. Furthermore, it is possible, with the appropriate configuration, lead length, static magnetic field strength, and other settings, to introduce resonant circuitry between 
the transmitted RF power and the lead. This could result in very rapid and clinically significant lead heating, especially at the lead tips, in a matter of seconds to a magnitude sufficient to result in tissue thermal injury or burns. This can also theoretically occur with implanted leads or wires even when they are not connected to any other device at either end. For illustration, the FDA has noted several reports of serious injury including coma and permanent neurological impairment in patients with implanted neurological stimulators who underwent MR imaging examinations. The injuries in these instances resulted from heating of the electrode tips $(19,20)$.

Furthermore, it is entirely possible for a lead or wire to demonstrate no significant heating while undergoing MR imaging examinations at 1.5 Tesla yet demonstrate clinically significant and potentially harmful degrees of heating within seconds at, for example, 3 Tesla. It has also been demonstrated that leads may demonstrate no significant heating at 3 Tesla yet may rapidly heat to hazardous levels when undergoing MR imaging at, for example, 1.5 Tesla. (Personal Observation, MR Safety testing, E. Kanal, MD, University of Pittsburgh Medical Center MR Research Center, 8/28/ 05) Thus at no time should a label of MR Conditional for thermal issues at a given field strength be applied to any field strength, higher or lower, other than the specific one at which safety was demonstrated.

Thus, exposure of electrically conductive leads or wires to the RF transmitted power during $\mathrm{MR}$ scanning should only be performed with caution and with appropriate steps taken to ensure significant lead or tissue heating does not result (see section H.9, below).

3. When electrically conductive materials external to the patient are required to be within the bore of the MR scanner with the patient during imaging, care should be taken to place thermal insulation (including air, pads, etc.) between the patient and the electrically conductive material, while simultaneously attempting to (as much as feasible) keep the electrical conductor from directly contacting the patient during imaging. It is also appropriate to try to position the leads or wires as far as possible from the inner walls of the MR scanner if the body coil is being used for RF transmission. When it is necessary that electrically conductive leads directly contact the patient during imaging, consideration should be given to prophylactic application of cold compresses or ice packs to such areas.

4. There have been rare reports of thermal injuries/ burns associated with clothing that contained electrically conductive materials, such as metallic threads, electrically conductive designs, and silver impregnated clothing. As such, consideration should be given to having all patients remove their own clothing and instead change into provided gowns to cover at the very least the region/ volume of the patient that is scheduled to undergo
MR imaging and, therefore, RF irradiation.

5. To help safeguard against thermal injuries or burns, depending on specific magnet designs, care may be needed to ensure that the patient's tissue(s) do not directly come into contact with the inner bore of the MR imager during the MRI process. This is especially important for several higher field MR scanners. The manufacturers of these devices provide pads and other such insulating devices for this purpose, and manufacturer guidelines should be strictly adhered to for these units.

6. It is important to ensure the patient's tissues do not form large conductive loops. Therefore, care should be taken to ensure that the patient's arms or legs are not positioned in such a way as to form a large caliber loop within the bore of the MR imager during the imaging process. For this reason, it is preferable that patients be instructed not to cross their arms or legs in the MR scanner. We are also aware of unpublished reports of thermal injury that seem to have been associated with skin-to-skin contact such as in the region of the inner thighs. It might be prudent to consider ensuring that skin-to-skin contact instances are minimized or eliminated in or near the regions undergoing radiofrequency energy irradiation.

7. Skin staples and superficial metallic sutures: Patients requested to undergo MR studies in whom there are skin staples or superficial metallic sutures (SMS) may be permitted to undergo the MR examination if the skin staples or SMS are not ferromagnetic and are not in or near the anatomic volume of RF power deposition for the study to be performed. If the nonferromagnetic skin staples or SMS are within the volume to be $\mathrm{RF}$ irradiated for the requested MR study, several precautions are recommended.

a. Warn the patient and make sure that they are especially aware of the possibility that they may experience warmth or even burning along the skin staple or SMS distribution. The patient should be instructed to report immediately if they experience warmth or burning sensations during the study (and not, for example, wait until the "end of the knocking noise").

b. It is recommended that a cold compress or ice pack be placed along the skin staples or SMS if this can be safely clinically accomplished during the MRI examination. This will help to serve as a heat sink for any focal power deposition that may occur, thus decreasing the likelihood of a clinically significant thermal injury or burn to adjacent tissue.

8. For patients with extensive or dark tattoos, including tattooed eyeliner, to decrease the potential for RF heating of the tattooed tissue, it is recommended that cold compresses or ice packs be placed on the tattooed areas and kept in place throughout the MRI process if these tattoos are within the volume in which the body coil is being used for RF transmission. This approach is 
especially appropriate if fast spin echo (or other high RF duty cycle) MRI sequences are anticipated in the study. If another coil is being used for RF transmission, a decision must be made if high RF transmitted power is to be anticipated by the study protocol design. If so, then the above precautions should be followed. Additionally, patients with tattoos that had been placed within $48 \mathrm{~h}$ before the pending MR examination should be advised of the potential for smearing or smudging of the edges of the freshly placed tattoo.

9. The unconscious or unresponsive patient should have all attached leads covered with a cold compress or ice pack at the lead attachment site for the duration of the MR study.

10. As noted above, it has been demonstrated that resonant circuitry can be established during MRI between the RF energies being transmitted and specific lengths of long electrically conductive wires or leads, which can thus act as efficient antennae. This can result in heating of the tips of these wires or leads to temperatures in excess of $90^{\circ} \mathrm{C}$ in a few seconds. Therefore, patients in whom there are long electrically conductive leads, such as Swan-Ganz thermodilution cardiac output capable catheters or Foley catheters with electrically conductive leads as well as electrically active implants containing leads such as pacemakers, ICDs, neurostimulators, and cochlear implants, let alone electrically active implant such as pacemakers, should be considered at risk for MR studies if the body coil is to be used for RF transmission over the region of the electrically conductive lead, even if only part of the lead pathway is within the volume to undergo RF irradiation. This is especially true for higher-field systems (e.g., greater than $0.5 \mathrm{~T}$ ) and for imaging protocols using fast spin echo or other high-RF duty cycle MRI sequences. Each such patient should be reviewed and cleared by an attending level 2 radiologist and a risk benefit ratio assessment performed before permitting them access to the MR scanner.

11. The potential to establish substantial heating is itself dependent upon multiple factors, including, among others, the static magnetic field strength of the MR scanner (as this determines the transmitted radiofrequencies ( $\mathrm{RF}$ ) at which the device operates) and the length, orientation, and inductance of the electrical conductor in the RF irradiated volume being studied. Virtually any lead lengths can produce substantial heating. Innumerable factors can affect the potential for tissue heating for any given lead. It is therefore critical to recognize that of all electrically conductive implants, it is specifically wires, or leads, that pose the greatest potential hazard for establishing substantial power deposition/heating considerations.

Another important consideration is that as a direct result of the above, it has already been demonstrated in vitro that heating of certain implants or wires may be clinically insignificant at, for example, 1.5 Tesla but quite significant at 3.0 Tesla. How- ever, it has also been demonstrated that specific implants might demonstrate no significant thermal issues or heating at 3.0 Tesla, but may heat to clinically significant or very significant levels in seconds at, for example, 1.5 Tesla. Thus, it is important to follow established product MR Conditional labeling and safety guidelines carefully and precisely, applying them to and only to the static magnetic field strengths at which they had been tested. MR scanning at either stronger and/or weaker magnetic field strengths than those tested may result in significant heating where none had been observed at the tested field strength(s).

\section{Drug Delivery Patches and Pads}

Some drug delivery patches contain metallic foil. Scanning the region of the metallic foil may result in thermal injury (21). Because removal or repositioning can result in altering of patient dose, consultation with the patient's prescribing physician would be indicated in assessing how to best manage the patient. If the metallic foil of the patch delivery system is positioned on the patient so that it is in the volume of excitation of the transmitting RF coil, the case should be specifically reviewed with the radiologist or physician covering the case. Alternative options may include placing an ice pack directly on the patch. This solution may still substantially alter the rate of delivery or absorption of the medication to the patient (and be less comfortable to the patient, as well). This ramification should therefore not be treated lightly, and a decision to proceed in this manner should be made by a knowledgeable radiologist attending the patient and with the concurrence of the referring physician as well.

If the patch is removed, a specific staff member should be given responsibility for ensuring that it is replaced or repositioned at the conclusion of the MR examination.

\section{J. Cryogen-Related Issues}

1. For superconducting systems, in the event of a system quench, it is imperative that all personnel and patients be evacuated from the MR scan room as quickly as safely feasible and the site access be immediately restricted to all individuals until the arrival of MR equipment service personnel. This is especially so if cryogenic gases are observed to have vented partially or completely into the scan room, as evidenced in part by the sudden appearance of white "clouds" or "fog" around or above the MR scanner. As noted in section B.3.n above, it is especially important to ensure that all police and fire response personnel are restricted from entering the MR scan room with their equipment (axes, air tanks, guns, etc.) until it can be confirmed that the magnetic field has been successfully dissipated, as there may still be considerable static magnetic field present despite a quench or partial quench 
of the magnet (22).

2 . It should be pointed out that room oxygen monitoring was discussed by the MR Blue Ribbon Panel and rejected at this time because the present oxygen monitoring technology was considered by industry experts not to be sufficiently reliable to allow for continued operation during situations of power outages, etc.

\section{K. Claustrophobia, Anxiety, Sedation, Analgesia and Anesthesia}

Adult and pediatric patient anxiolysis, sedation, analgesia, and anesthesia for any reason should follow established ACR (23), American Society of Anesthesiologists (ASA) (15,24-26), and TJC standards (17).

\section{Contrast Agent Safety}

\section{Contrast agent administration issues:}

No patient is to be administered prescription MR contrast agents without orders from a duly licensed physician. Intravenous injection-qualified MR technologists may start and attend to peripheral IV access lines if they have undergone the requisite site-specified training in peripheral IV access and have demonstrated and documented appropriate proficiency in this area. IV-qualified MR technologists may administer FDA-approved gadolinium-based MR contrast agents by means of peripheral IV routes as a bolus or slow or continuous injection as directed by the orders of a duly licensed site physician.

Administration of these agents is to be performed as per the ACR policy. The ACR approves of the injection of contrast material and diagnostic levels of radiopharmaceuticals by certified and/or licensed radiologic technologists and radiologic nurses under the direction of a radiologist or his or her physician designee who is personally and immediately available, if the practice is in compliance with institutional and state regulations. There must also be prior written approval by the medical director of the radiology department/service of such individuals. Such approval process must follow established policies and procedures, and the radiologic technologists and nurses who have been so approved must maintain documentation of continuing medical education related to materials injected and to the procedures being performed (27).

The name of the administered contrast agent, the administered dose, and the route (and, if applicable, rate) of administration as well as any adverse reactions, if any, should be recorded for all contrast agents administered as part of the executed MR examination.

\section{Prior Contrast Agent Reaction Issues:}

a. According to the ACR Manual on Contrast Media (28) adverse events after intravenous injection of gadolinium seem to be more common in patients who had previous reactions to an MR contrast agent. In one study, $16(21 \%)$ of 75 patients who had previous adverse reactions to MR contrast agents reacted to subsequent injections of gadolinium. Patients with asthma also seem to be more likely to have an adverse reaction to administration of a gadolinium-based MR contrast agent. Patients with allergies also seemed to be at increased risk ( 2.0-3.7 times, compared with patients without allergies). Patients who have had adverse reactions to iodinated contrast media are more than twice as likely to have an adverse reaction to gadolinium (6.3\% of 857 patients).

b. At present, there are no well-defined policies for patients who are considered to be at increased risk for having adverse reaction to MR contrast agents. However, the following recommendations are suggested: patients who have previously reacted to one MR contrast agent can be injected with another agent if they are restudied, and at-risk patients can be premedicated with corticosteroids and, occasionally, antihistamines.

c. All patients with asthma, allergic respiratory histories, prior iodinated or gadolinium-based contrast reactions, etc. should be followed more closely as they are at a demonstrably higher risk of adverse reaction.

\section{Severe Renal Disease, Gadolinium-Based MR} Contrast Agents, and Nephrogenic Fibrosing Dermopathy/Nephrogenic Systemic Fibrosis (NFD/NSF)

Since the prior version of this document the ACR decided that MR safety issues related to gadolinium based contrast agents (GBCA) and NSF would be the purview of the ACR Committee on Drugs and Contrast Media. For the recommendations of the ACR Committee on Drugs and Contrast Media regarding GBCA and NSF, the reader is referred to the most recent publication of that committee in this regard which appears in the ACR Manual on Contrast Media, Version 8, Chapter 13, Nephrogenic Systemic Fibrosis. The most recent version of the ACR Manual on Contrast Media may be downloaded from the American College of Radiology website at http://www.acr.org/ Quality-Safety/Resources.

\section{Patients in Whom There Are or May Be Intracranial Aneurysm Clips}

1. In the event that it is unclear whether a patient does or does not have an aneurysm clip in place, plain films should be obtained. Alternatively, if available, any cranial plain films, CT or MR examination that may have taken in the recent past (i.e., subsequent to the suspected surgical date) should be reviewed to assess for a possible intracranial aneurysm clip.

2. In the event that a patient is identified to have an intracranial aneurysm clip in place, the MR examination should not be performed until it can be documented that the specific manufacturer, 
model, and type of aneurysm clip within that patient is MR Safe or MR Conditional. All documentation of types of implanted clips, dates, etc. must be in writing and signed by a licensed physician. Phone or verbal histories and histories provided by a nonphysician are not acceptable. Fax copies of operative reports, physician statements, etc. are acceptable as long as a legible physician signature accompanies the requisite documentation. A written history of the clip itself having been appropriately tested for ferromagnetic properties (and description of the testing methodology used) before implantation by the operating surgeon is also considered acceptable if the testing follows the standard test methods established by ASTM International.

3. All intracranial aneurysm clips manufactured 1995 or later for which the manufacturer's product labeling continues to claim MR Conditional labeling may be accepted for MR scanning without further testing.

4. Clips manufactured before 1995 require either pretesting (as per the ASTM F2503 standard practice guidelines) before implantation or individual review of previous MRI of the clip or brain in that particular case, if available. By assessing the size of the artifact associated with the clip relative to the static field strength on which it was studied, the sequence type, and the MRI parameters selected, an opinion may be issued by one of the site's level $2 \mathrm{MR}$ attending radiologists as to whether the clip demonstrates significant ferromagnetic properties or not. Access to the MR scanner would then be based on that opinion.

5. A patient with an aneurysm clip (or other implant) may have safely undergone a prior MR examination at any given static magnetic field strength. This fact in and of itself is not sufficient evidence of the implant's MR safety and should not solely be relied upon to determine the MR safety or compatibility status of that aneurysm clip (or other implant).

Variations in static magnetic field strength, static magnetic field spatial gradient, orientation of the aneurysm clip (or other implant) to the static magnetic field or static field gradient, rate of motion through the spatial static field gradient, etc. are all variables that are virtually impossible to control or reproduce. These variables may not have resulted in adverse event in one circumstance but may result in significant injury or death on a subsequent exposure. For example, a patient who went blind from interactions between the metallic foreign body in his retina and the spatial static fields of the MR scanner entered the magnet and underwent the entire MR examination without difficulty; he only went blind upon exiting the MR scanner at the completion of the examination.

6. Barring availability of either pretesting or prior MRI data of the clip in question, a risk-benefit assessment and review must be performed in each case individually. Furthermore, for patients with intracranial clips with no available ferromagnetic or imaging data, should the riskbenefit ratio favor the performance of the MR study, the patient or guardian should provide written informed consent that includes death as a potential risk of the MRI procedure before permitting that patient to undergo an MR examination.

\section{N. Patients in Whom There are or May Be Cardiac Pacemakers or Implantable Cardioverter Defibrillators}

MRI of Cardiac Implantable Devices

Background: Cardiac implantable electronic devices (CIEDs) have expanded in number and complexity since their introduction in 1958 and now include cardiac pacemakers, implantable cardioverter-defibrillators (ICD), implantable cardiovascular monitors (ICM) and implantable loop recorders (ILR). Pacemakers (pulse generators) and leads that are FDA labeled MR conditional became available in the U.S., February of 2011 and both commercially available ILRs are labeled also "MR Conditional" The vast majority of CIEDs, however, are not labeled as MR Conditional. No ICDs are currently labeled "MR Conditional" and none are expected to be clinically available for several years. Patient product wallet identification cards, industry maintained databases, plain film lead and pulse generator X-ray identifiers, and operative notes may assist in identification of MR Conditional patient hardware.

Radiologists and cardiovascular specialists must be familiar with restrictions for each device, recognizing that because each MR conditional device is unique, there are no "universal" labeling guidelines that are applicable for all. Failure to follow the product labeling for a particular device is "off-label" and could result in an adverse event.

Potential Complications: Unexpected programming changes, inhibition of pacemaker output, failure to pace, transient asynchronous pacing, rapid cardiac pacing, the induction of ventricular fibrillation, heating of the tissue adjacent to the pacing or ICD system and especially cardiac tissue near the lead tip, early battery depletion, and outright device failure requiring replacement may occur during MRI of patients with pacemakers or ICDs $(18,29-31)$. Multiple deaths have been documented to occur under poorly and incompletely characterized circumstances when CIED patients underwent MRI (32-34). These deaths may have occurred as a result of pacemaker inhibition, failure to capture or device failure (resulting in prolonged asystole) and or rapid cardiac pacing or asynchronous pacing (resulting in the initiation of ventricular tachycardia or fibrillation).

Patient Assessment: Most CIEDs can be viewed as having a pacing or defibrillator "system" comprised of a pulse generator and one or more leads. Current insertable loop recorders are leadless and are for monitoring (not therapeutic) purposes alone. 
Importantly, leads and occasionally pulse generators may have been "abandoned" in the patient from previous implants. As such, each patient may have a "system" that includes both active and inactive (abandoned) hardware. While electrically and therapeutically inactive with regard to pacing functionality outside the MR suite, abandoned hardware may pose a substantial risk if exposed to MRI energies irrespective of the MR compatibility of the active pacing hardware (35). Indeed, abandoned leads may well pose a greater relative risk of lead tip heating if exposed to the MR imaging process than leads that are part of an actively implanted system.

The experience with MR imaging in patients who have retained metallic materials after cardiac surgery such as epicardial pacing leads is perhaps helpful (36). While some have produced survey data suggesting that in the case of postoperatively retained cardiac pacing wires, "the absence of reported complications in thousands of exposed patients suggests that the risk is low (37), others have voiced appropriate concern as to the general relevance of this data to the overall population (38)."

\section{Patient Assessment for MRI}

All device hardware should be included in the practitioner's assessment of the patient's suitability for MR scanning. Coordination with the physician managing the device (cardiologist/electrophysiologist) and a representative from the device manufacturer is of paramount importance to determine whether the system (pulse generator and leads) is MR Conditional. Practitioners and their staff should note that the entire system (pulse generator and leads) must be labeled "MR Conditional" for a system to in fact be considered MR conditionally safe. Furthermore, an MR Conditional system is only considered safe if all of the MR conditions for safe use are followed. The presence of abandoned leads from previous nonlabeled systems or "mix-and-match" systems (combined MR Conditional labeled and nonlabeled hardware) renders the system as a whole MR Unsafe or at best "MR unknown". Importantly, because of the potential for heating, an abandoned (unattached to a pulse generator) MR Conditional lead should be considered, and from a risk assessment point of view, treated as, MR Unsafe.

The patient's attestations as to their device MR compatibility is not sufficient to establish MR safety. To provide for the safest scanning experience, to minimize confusion and disappointment, to prevent delays in diagnosis due to rescheduling, and to limit the potential for throughput disruption in an already busy MR schedule, we recommend the development of institutional policies, protocols and care pathways for all patients with implantable rhythm devices irrespective of their labeling. Careful, thoughtful advance planning and close collaboration between the radiology and cardiology staffs and the industry representatives of the device manufacturers will provide the greatest likelihood for a consistent, safe experience.
Unlabeled Cardiac Devices: Amongst the patients with MR unsafe CIEDs, many have conditions that would ordinarily be assessed with MRI. While many can have their medical conditions managed without MRI, in some instances, specific clinical circumstances may present compelling reasons for undergoing an MR examination (39). Should MRI be considered, it should be evaluated on a case-by-case and site-bysite basis and only if the site is manned with individuals with the appropriate radiology and cardiology knowledge and expertise on hand.

The committee eschews the term "modern" when referring to a particular device, recognizing that all devices not labeled for use in the MRI contain legacy components and designs that may not be resistant to the forces and electromagnetic interference present in the MRI suite. All devices, unless appropriately tested and labeled, should never be regarded as safe for MRI simply because they are "modern" or recently manufactured.

Consent: The patient with a pacemaker or ICD that is not labeled as MR Conditional should be apprised of the risks associated with MRI and should provide informed consent. While the majority of reported deliberate scans of device patients have proceeded without adverse effects when appropriate precautions were undertaken, several have not, and there is under-reporting of adverse events, including deaths. Thus, assignment of a risk benefit ratio to the performance of MRI in any given device patient is difficult. While the risk may be low, patients with devices that are not labeled as MR Conditional should be advised that life-threatening arrhythmias might occur during MRI and serious device malfunction might occur requiring replacement of the device.

Precautions during MRI with CIEDs: Should any MRI examination be contemplated for a patient with an implanted pacemaker or ICD, it is recommended that radiology and cardiology personnel and a fully stocked crash cart be readily available throughout the procedure in case a significant arrhythmia develops during the examination that does not terminate with the cessation of the MR study. The cardiologist should be familiar with the patient's arrhythmia history and the implanted device. A programmer that can be used to adjust the device should be readily available. The goal of pre-MRI programming should be to mitigate the risk to the patient and the device while undergoing MRI $(40,41)$. All such patients should be actively monitored throughout the examination. A central monitoring facility located in the hospital with appropriately trained staff may be sufficient to monitor appropriately selected low risk device patients undergoing MR scanning.

At a minimum, EKG and pulse oximetry should be used for monitoring these patients. At the conclusion of the examination, the device should be interrogated to confirm that the function is consistent with the pre-examination state. In the absence of detected post MR anomalies, the value of repeating device re-evaluation is controversial. However, the clinician may recommend a post-scan follow-up check of the patient's device (1-6 weeks) following the scan to 
confirm appropriate function. For appropriately selected patients who have no post-MRI device abnormalities demonstrated, remote follow-up through home monitoring seems appropriate. There is no compelling evidence that post MRI defibrillation threshold testing is required if the MR-exposed ICD shows no post MRI anomalies (42).

Should an MRI (or entry into the magnet area) be performed inadvertently on a patient with a pacemaker or ICD, the patient's cardiologist should be contacted before the patient's discharge from the MRI suite. Exposure to the static magnetic field alone may adversely affect device function or alter its programming (43-45). The importance of interrogation of the device before the patient's leaving the MRI suite cannot be overstated.

\section{APPENDIX 1}

\section{Personnel Definitions}

Non-MR Personnel

Patients, visitors or facility staff who do not meet the criteria of level 1 or level 2 MR personnel will be referred to as non-MR personnel. Specifically, non-MR personnel will be the terminology used to refer to any individual or group who has not within the previous 12 months undergone the designated formal training in MR safety issues defined by the MR safety director of that installation.

\section{Level 1 MR Personnel}

Individuals who have passed minimal safety educational efforts to ensure their own safety as they work within Zone III will be referred to as level 1 MR personnel (e.g., MRI department office staff, and patient aides.)

\section{Level 2 MR Personnel}

Individuals who have been more extensively trained and educated in the broader aspects of MR safety issues, including, issues related to the potential for thermal loading or burns and direct neuromuscular excitation from rapidly changing gradients, will be referred to as level $2 \mathrm{MR}$ personnel (e.g., MRI technologists, radiologists, radiology department nursing staff.)

\section{ZONE DEFINITIONS}

\section{Zone I}

This region includes all areas that are freely accessible to the general public. This area is typically outside the MR environment itself and is the area through which patients, health care personnel, and other employees of the MR site access the MR environment.

\section{Zone II}

This area is the interface between the publicly accessible uncontrolled Zone I and the strictly controlled Zone III (see below). Typically, the patients are greeted in Zone II and are not free to move throughout Zone II at will, but rather are under the supervision of MR personnel. It is in Zone II that the answers to MR screening questions, patient histories, medical insurance questions, etc. are typically obtained.

\section{Zone III}

This area is the region in which free access by unscreened non-MR personnel or ferromagnetic objects or equipment can result in serious injury or death as a result of interactions between the individuals or equipment and the MR scanner's particular environment. These interactions include, but are not limited to, those with the MR scanner's static and time varying magnetic fields. All access to Zone III is to be strictly physically restricted, with access to regions within it (including Zone IV; see below) controlled by, and entirely under the supervision of, MR personnel.

\section{Zone IV}

This area is synonymous with the MR scanner magnet room itself. Zone IV, by definition, will always be located within Zone III as it is the MR magnet and its associated magnetic field which generates the existence of Zone III.

Non-MR Personnel should be accompanied by, or under the immediate supervision of and visual contact with, one specifically identified level 2 MR person for the entirety of their duration within Zone III or IV restricted regions.

Level 1 and 2 MR personnel may move freely about all zones. 
APPENDIX 2

Safety Screening Form for Magnetic Resonance (MR) Procedures

Date Name (first middle last)

Female [ ] Male [ ]

Age

Date of Birth

Height

Weight

Why are you having this examination (medical problem)?

Have you ever had an MRI examination before and had a problem? If yes, please describe

Have you ever had a surgical operation or procedure of any kind? If yes, list all prior surgeries and approximate dates:

Have you ever been injured by a metal object or foreign body (e.g., bullet, BB shrapnel)? If yes, please describe

Have you ever had an injury from a metal object in your eye (metal slivers, metal shavings, other metal object)?

If yes, did you seek medical attention?

If yes, describe what was found

Do you have a history of kidney disease, asthma, or other allergic respiratory disease?

Do you have any drug allergies?

If yes, please list drugs

Have you ever received a contrast agent or X-ray dye used for MRI, CT, or other X-ray or study?

Have you ever had an X-ray dye or magnetic resonance imaging (MRI) contrast agent allergic reaction? If yes, please describe

Are you pregnant or suspect you may be pregnant?

Are you breast feeding?

Date of last menstrual period Post-menopausal? 


\section{MR Hazard Checklist}

Please mark on the drawings provided the location of any metal inside your body or site of surgical operation.

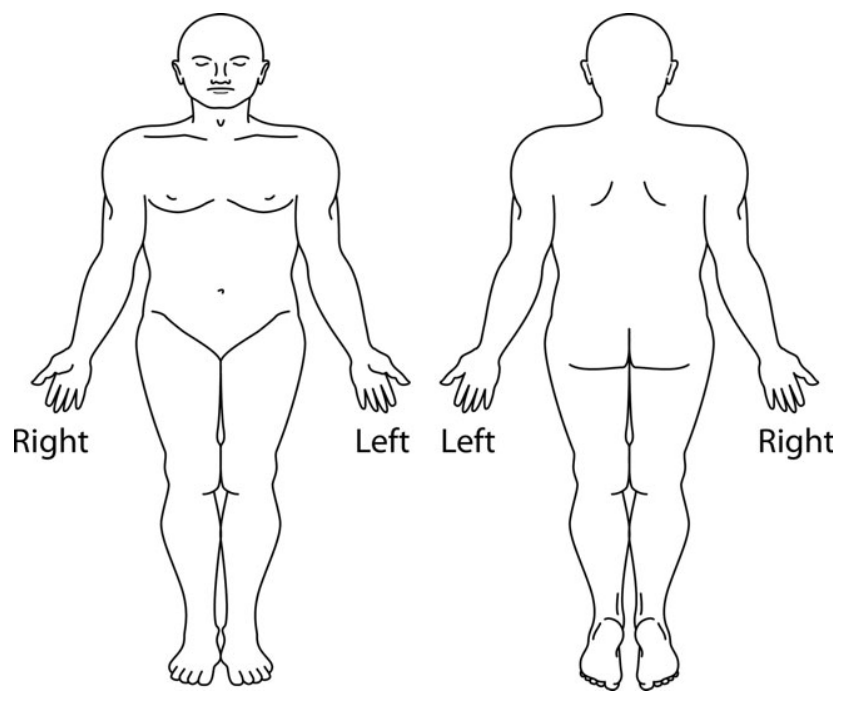

The following items may be harmful to you during your MR scan or may interfere with the MR examination. You must provide a "yes" or "no" for every item. Please indicate if you have or have had any of the following:

YES NO

Any type of electronic, mechanical, or magnetic implant

Type

Cardiac pacemaker

Aneurysm clip

Implanted cardiac defibrillator

Neurostimulator

Biostimulator

Type

Any type of internal electrodes or wires

Cochlear implant

Hearing aid

Implanted drug pump (e.g., insulin, Baclofen, chemotherapy, pain medicine)

Halo vest

Spinal fixation device

Spinal fusion procedure

Any type of coil, filter, or stent

Type 
Any type of metal object (e.g., shrapnel, bullet, BB)

Artificial heart valve

Any type of ear implant

Penile implant

Artificial eye

Eyelid spring

Any type of implant held in place by a magnet

Type

Any type of surgical clip or staple

Any IV access port (e.g., Broviac, Port-a-Cath, Hickman, Picc line)

Medication patch (e.g., Nitroglycerine, nicotine)

Shunt

Artificial limb or joint

What and where

Tissue Expander (e.g., breast)

Removable dentures, false teeth or partial plate

Diaphragm, IUD, Pessary

Type

Surgical mesh

Location

Body piercing

Location

Wig, hair implants

Tattoos or tattooed eyeliner

Radiation seeds (e.g., cancer treatment)

Any implanted items (e.g., pins, rods, screws, nails, plates, wires)

Any hair accessories (e.g., bobby pins, barrettes, clips)

Jewelry

Any other type of implanted item

Type 


\section{Instructions for the Patients}

1. You are urged to use the ear plugs or headphones that we supply for use during your MRI examination because some patients may find the noise levels unacceptable, and the noise levels may affect your hearing.

2. Remove all jewelry (e.g., necklaces, pins, rings).

3. Remove all hair pins, bobby pins, barrettes, clips, etc.

4. Remove all dentures, false teeth, partial dental plates.

5. Remove hearing aids.

6. Remove eyeglasses.

7. Remove your watch, pager, cell phone, credit and bank cards and all other cards with a magnetic strip.

8. Remove body piercing objects.

9. Use gown, if provided, or remove all clothing with metal fasteners, zippers, etc.

I attest that the above information is correct to the best of my knowledge. I have read and understand the entire contents of this form, and I have had the opportunity to ask questions regarding the information on this form.

Patient signature

$\mathrm{MD} / \mathrm{RN} / \mathrm{RT}$ signature

Date

Print name of MD, RN, RT

For MRI Office Use Only

Patient Name

Patient ID Number

Procedure

Clinical History

\section{Hazard Checklist for MRI Personnel}

YES

$$
\text { NO }
$$

Endotracheal tube

Swan-Ganz catheter

Extra ventricular device

Arterial line transducer

Foley catheter with temperature sensor and/or metal clamp

Rectal probe

Esophageal Probe

Tracheotomy tube

Guidewires
Referring Physician

Diagnosis 


\section{APPENDIX 3}

\section{MR Facility Safety Design Guidelines}

The goal of MR safety is to prevent harm to patients, though a MR facility cannot simply adopt one or two interventions and hope to successfully attain this objective. According to safety and human factors engineering principles, multiple safety strategies must be adopted to be effective. This approach is sometimes termed "defense in depth." The safety strategies outlined in the main body of this MR Safe Practice Guidelines document include, for instance, policies that restrict personnel access, specialized training and drills for MR personnel, and warning labels for devices to be brought into Zone IV regions.

Along with these people-oriented strategies of policies and training, organizations need also to adopt the strategies of safety-oriented architectural and interior design. These design elements can support the other safety strategies, by making them easier or more obvious to follow. The architectural enhancements described herein add one or more strong barriers to enhance "defense in depth".

This appendix includes descriptions of architectural and interior design recommendations organized around the many MR suite functional areas. Note that a facility's design can encourage safety and best practices by improving the flow of patients, various healthcare personnel, and equipment and devices, and not just by to preventing MR unsafe items from becoming missiles, or screening out patients with hazardous implanted devices.

Placing design elements strategically in a suite layout such that the element supports best practice workflow patterns will increase compliance with safer practices. For example, having a private area for patient screening interviews will make it more likely the patients will disclose sensitive types of implants. Another example of designing for safety is to include dedicated space and temporary storage for MR Unsafe equipment (e.g., ferromagnetic IV poles, transport stretchers) out of direct sight and away from people flow patterns.

Effective and safe MRI suites must balance the technical demands of the MR equipment with local and state building codes, standards of accrediting bodies, clinical and patient population needs, payor requirements and a collage of civil requirements from Health Insurance Portability and Accountability Act (HIPAA) to the Americans with Disabilities Act (ADA).

In an effort to better match appropriate facility design guidelines with levels of patient acuity and care, the ACR MR Safety Committee is currently developing level designations for MRI facilities in conjunction with the efforts of Committees from other Societies and Organizations. These will address customization of requirements for sites with varying anticipated patient care sedation, anesthesia, and/or interventional activities.

While it would be desirable to provide a universal MRI suite safety design, the variables are too numerous to adequately address in a single template. The following MRI Facility Safety Design Guidelines are provided to provide information in support of planning, design and construction of MR facilities, including updates to existing MR facilities, which enhance the safety of patients, visitors and staff. This information is intended to supplement and expand upon patient safety guidance provided throughout the ACR MR Safe Practice Guidelines document.

\section{MR Equipment Vendor Templates}

Design templates provided by MR equipment manufacturers are invaluable in developing suites that meet the minimum technical siting requirements for the specific equipment. Vendor design templates, however, typically depict only the control and equipment rooms, in addition to the magnet room, Zone IV.

Patient/family waiting, interview areas, physical screening/changing areas, access controls, storage, crash carts, induction, medical gas services, postscreened patient holding areas, infection control provisions, and interventional applications, among many other issues, are not addressed in typical vendor provided drawings. These issues are left to facility owners, operators and their design professionals to resolve. The guidance which follows is designed to address many of these issues which directly impact safety within the MR suite.

\section{Patient interview/clinical screening areas (Zone II)}

Reviewing the patient Safety Screening Form and MR Hazard Checklist requires discussing confidential personal information. To facilitate full and complete patient disclosure of their medical history, this clinical screening should be conducted in an area which provides auditory and visual privacy for the patient. Facilities should prospectively plan for electronic patient medical records, which are useful in clinical screening, and should provide for access to records in the MR suite in support of clinical patient screening.

Clinical screening of inpatients may be completed in the patient room for hospital-based MR facilities. However, all screenings are to be double-checked and verified by appropriately trained MR Personnel before MR examination.

\section{Physical screening and patient changing/gowning rooms (Zone II)}

All persons and objects entering Zone III should be physically screened for the presence of ferromagnetic materials which, irrespective of size, can become threats in proximity to the MR. A location should be provided for patients in which they may change out of their street clothes and into a facility provided gown or scrubs, if/as deemed appropriate. For those facilities which either do not provide space for, or do not require, patient changing, the facility must provide alternative means of identifying and removing items which the patient may have brought with them that might pose threats in the MR environment.

A high-strength hand-held magnet is a recommended tool to evaluate the gross magnetic characteristics of objects of unknown composition. Magnetic 
strength for these permanent magnets fall off quickly as one moves away from the face of the magnet. Thus, these may not demonstrate attraction for ferromagnetic components which are not superficially located or cannot for whatever reason be brought into close proximity with the surface of this hand-held magnet.

Ferromagnetic detection systems have been demonstrated to be highly effective as a quality assurance tool, verifying the successful screen and identifying ferromagnetic objects which were not discovered by conventional screening methods. It is recommended that new facility construction anticipate the use of ferromagnetic detection screening in Zone II and provide for installation of the devices in a location which facilitates use and throughput. Many current ferromagnetic detection devices are capable of being positioned within Zone III, even at the door to the magnet room, however a recommended use of ferromagnetic detection is to verify the screening of patients before passing through the controlled point of access into Zone III.

Physical screening of patients should consist of removal of all jewelry, metallic/ferromagnetic objects, onplants and prostheses (as indicated by manufacturer's conditional use requirements and physician instructions), and either having patients change out of their street clothes into facility provided gowns/ scrubs or thorough screening of street clothes, including identifying the contents of pockets and composition of metallic fibers, fasteners, and reinforcing.

While gowning maybe helpful, it is certainly not fool proof in precluding a patient from entering with ferromagnetic material on them.

\section{Transfer Area/Ferrous Quarantine Storage (Zone II)}

Patients arriving with wheelchairs, walkers, portable oxygen and other appliances that may be unsafe in the MR environment should be provided by the facility with appropriate MR safe or MR conditional appliances. An area should be provided to transfer the patient from unsafe appliances to ones appropriate to the MR environment. Unsafe appliances brought by the patient should be secured in a 'ferrous quarantine' storage area, distinct from storage areas for MR safe and MR conditional equipment and ideally locked out of sight. Patient belongings should be retrieved from the 'ferrous quarantine' only when discharging the patient to whom the objects belong from the MR suite.

\section{Access control (Zone III/Zone IV)}

Means of physically securing and restricting access to Zone III from all adjacent areas must be provided. Independent access into Zone III must be limited to only appropriately trained MR personnel.

\section{Patient Holding (Zone III)}

Depending upon facility capacity and patient volume, it may be advisable to provide a postscreened patient holding area. Zone III holding areas should be equipped and appointed to prevent patient exit and subsequent re-entry. This will help prevent the inadvertent-or even intentional-introduction of unscreened objects and personnel.

Many multi-modal radiology facilities combine patient holding and/or induction areas for patients of different modalities. This presents safety challenges when, for example, patients scheduled to receive a CT are held in a patient holding area shared by postscreened MR patients. As CT patients would not typically be screened for MR contraindications or ferrous materials, this poses risks to both the CT patient with a contraindicated implant and to those in the MRI zone IV should an unscreened individual inadvertently enter with a ferrous object or implant.

Unless all persons in patient holding areas used for postscreened MR patients are screened for MRI, the practice of shared patient holding areas between MR and other modalities is discouraged. Ultimately it is the responsibility of trained MR staff to verify the screening of any co-mingled patient before permitting them access to Zone III and Zone IV.

In all MR facilities, Zone III is required to be physically secured and access limited to only MR personnel and successfully MR prescreened non-MR personnel accompanied by MR personnel. Ideally, facilities should be designed such that patients for other modalities are not co-mingled with postscreened MR patients.

\section{Lines of Sight/Situational Awareness (Zone III)}

Trained MR personnel are arguably the single greatest safety resource of MR facilities. These individuals should be afforded visual control over all persons entering or exiting Zones III or IV. The technologist seated at the MR operator console should therefore be able to view not only the patient in the MR scanner but also the approach and entrance into Zone IV. Line of sight between the MR system operator console and both the Zone IV entrance(s) and the patient within the MR scanner are requirements of the 2010 edition of Guidelines for Design and Construction of Health Care Facilities (46). When practical, suites should also be prospectively designed to provide a view from the MR operator's console to patient holding areas. If this cannot be satisfactorily achieved by direct line of sight, remote video viewing devices are an acceptable substitute toward accomplishing this objective.

The technologist at the console should also be provided with a view to induction/recovery areas within the MR suite, as applicable.

\section{Emergency Resuscitation Equipment (Zone II or Zone III)}

Because of risks associated with contrast agents, sedation, anesthesia, and even the frail health of patients undergoing MR examinations, it is advised that each facility have appropriate provisions for stabilization and resuscitation of patients.

It is recommended that crash carts and emergency resuscitation equipment be stored in a readily accessible area within either Zone II or Zone III. This emergency resuscitation equipment is to be appropriately 
labeled and also tested and verified as safe for usage in the MR environment for the anticipated conditions of usage.

MR facilities should maintain a supply of emergency medications to treat adverse reactions to administered contrast agents.

MR facilities providing care to patients who require clinical support during the MR examination should have emergency response equipment and personnel, trained in MR safety issues as well as trained to respond to anticipatable adverse events, readily available to respond to patient adverse events or distress in the MR arena.

\section{Fringe Magnetic Field Hazards (Zone III)}

For many MR system installations, magnetic fringe fields which project beyond the confines of the magnet room superimpose potential hazards on spaces which may be outside the MR suite, potentially on levels above or below the MR site and perhaps even outside the building. Facilities must identify all occupy-able areas, including those outside the MR suite (including rooftops, storage areas, mechanical closets, etc.) which are exposed to potentially hazardous magnetic fringe field strengths. Areas of potential hazard must be clearly identified and access to these areas restricted, just as they would be within the MR suite.

\section{Cryogen Safety (Zone IV)}

Liquid helium and liquid nitrogen represent the most commonly used cryogens in MR environments. The physical properties of these cryogenic liquids present significant potential safety hazards. If exposed to room air these cryogenic liquids will rapidly boil off and expand into a gaseous state. This produces several potential safety concerns, including:

- Asphyxiation potential as cryogenic gases replace oxygenated air.

- Frostbite considerations at the exceedingly low temperatures of these cryogenic liquids.

- Fire hazards can exist in the unlikely event of a quench, especially if some of the cryogenic gases escape into the magnet room/Zone IV

- Pressure considerations within Zone IV can rarely exist in the unlikely event of a quench in which some of the cryogenic gases escape into the magnet room/Zone IV.

a. Cryogen Fills. Though contemporary superconducting magnets require cryogen re-fills only infrequently, there is still almost always the need to periodically bring hundreds of liters of liquid cryogen to the magnet. It is because of the risks to persons near the magnet and storage/transport dewars that trans-fill operations should be undertaken with great care and only by appropriately trained personnel.

- Dewars containing cryogenic liquids should never be stored inside an MRI facility or indeed in any enclosed facility unless it is in a facility specifically designed to obviate the associated pressure, temperature, and asphyxiation risks.
- A cryogen transfill should never be attempted by untrained personnel or even with any unnecessary personnel in attendance, including MR personnel staff and patients, within Zone IV.

- Cryogen transfills should only be performed with appropriate precautions in place to prevent against pressure entrapment and asphyxiation.

b. Magnet Room Cryogen Safety. For most MRI systems if the magnet quenches, the escaping cryogenic gases are ducted outside the building to an unoccupied discharge area. However, there have been documented failures of cryogen vent/quench pipe assemblies which have led to considerable quantities of cryogenic gases being inadvertently discharged into the magnet room/Zone IV. The thermal expansion of the cryogens, if released into the magnet room, can positively pressurize the magnet room and entrap persons inside until such time that the pressure is equalized.

The following recommended MRI suite design and construction elements reduce patient and staff risks in the unlikely event of a quench in which the cryogen vent pathway (quench pipe) ruptures or leaks into Zone IV:

- All magnet rooms/Zone IV regions for superconducting magnets should be provided with an emergency exhaust pathway. The emergency exhaust grille is to be located in the ceiling opposite the entrance to the magnet room (Zone IV) door. At this location, when activated in the unlikely event of a quench breach, the exhaust fan is positioned to draw the vaporous cloud of cryogenic gas away from the door exiting from the magnet room.

- Many MR manufacturers are now requiring that magnet rooms for superconducting magnets also be provided with an additional form of passive pressure relief/pressure equalization to minimize the risks of positive-pressure entrapment. Designs for passive pressure relief mechanisms should follow design criteria similar to that of cryogen vent pathway and active exhaust, including discharge to a protected area as described in section 10.c below.

Some MR facilities are constructed without open waveguides or glass observation windows to Zone IV regions. In these facilities the potential risks of entrapment are even greater and may warrant an additional degree of attention in this regard.

While it can provide a degree of redundancy, it should be noted that, even with an exhaust fan, designing the door to Zone IV to swing outward is not, by itself, an appropriate means of pressure relief. In a severe positive pressure situation unlatching an outward-swinging door might permit the door to burst open with tremendous pressure, potentially injuring person(s) opening the door. If used as the only means of pressure equalization, an outward-swinging door may actually introduce new hazards to any staff person attempting to open the door to a pressurized magnet room from the outside. 
Similarly, though it has proven effective in lifethreatening situations, breaking a control window should not be advocated as a primary means of relieving/equalizing Zone IV pressure in a quench situation. It should be noted that the current construction of many RF shielded observation windows is such that it would make breaking the window very difficult, further diminishing it as a viable means of timely pressure relief.

Once provided with appropriate pressure equalization and emergency exhaust, magnet room door swing direction and design should be left to the discretion of a facility and their design professionals.

c. Cryogen Vent Pathway. Obstructions, inappropriate pipe materials, insufficient pipe caliber and/or length, or faulty connections in the length of the cryogen vent pathway can cause failure between the magnet and point of discharge. An evaluation of the current cryogen vent piping/ducting assembly is recommended to help identify and correct potential weaknesses that could potentially fail in a quench. Facilities are advised to evaluate the design and inspect the construction of their cryogen vent system.

Because minimum design requirements for some cryogen vent systems have been revised by magnet system vendors, facilities should obtain current standards from the original equipment manufacturers to use in evaluating their cryogen vent assembly and not rely on original siting requirements.

Beyond the assessment of the current construction of the cryogen vent system, it is prudent for MRI facilities:

- To inspect cryogen vent systems at least annually, identifying stress/wear of pipe sections and couplings, loose fittings and supports or signs of condensation/water within the cryogen vent pathway which may indicate a blockage.

- Following any quench of a superconducting magnet, to conduct a thorough inspection of cryogen vent system, including pipe sections, fittings, couplings, hangers and clamps, before returning the magnet to service.

Because obstructions/occlusions of the cryogen vent can increase the likelihood of rupture in a quench event, facilities should ensure that:

- The discharge point has an appropriate weatherhead which prevents horizontal, wind-driven precipitation from entering, collecting, or freezing in the quench exhaust pipe.

- The discharge point is high enough off of the roof or ground surface that snow or debris cannot enter or occlude the pipe.

- The discharge is covered by a material of sufficiently small openings to prevent birds or other animals from entering the quench pipe, while not occluding cryogenic gaseous egress in a quench situation.

Facilities that discover failings in any of these basic protections of the cryogen discharge point should immediately take additional steps to verify the patency of the cryogen vent and provide the minimum current discharge protections recommended by the original equipment manufacturer.
To protect persons from cryogen exposure at the point of discharge:

- At the point of cryogen discharge, a quench safety exclusion zone with a minimum clear radius of 25 feet ( 8 meters) should be established and clearly marked with surface warnings and signage.

- The quench safety exclusion zone should be devoid of serviceable equipment, air intakes, operable windows or unsecured doors that either require servicing or offer a pathway for cryogenic gasses to re-enter the building.

- Persons who must enter this quench safety exclusion zone, including incidental maintenance personnel and contractors, should be permitted to do so only after receiving specific instruction on quench risks and response.

\section{MR Conditional Devices (Zone IV)}

The normal or safe operation of many medical devices designed for use in the MR environment may be disrupted by exposure to conditions exceeding the device's conditional rating threshold. It is advisable for MR facilities to identify the allowable conditional rating for static field and spatial gradient exposure for each MR Conditional device which may be brought into Zones III and IV. MR Conditional devices may be conditionally safe for one specific field strength, but unsafe at higher or lower field strength. For prospective installations it is recommended that the location of critical isogauss line(s) be identified for MR Conditional equipment and devices used within the MR suite and delineated on the floor and walls of the magnet room to aid in the positioning and safe and effective operation of said equipment.

All MR facilities should evaluate all MR Conditional patient monitoring, ventilators, medication pumps, anesthesia machines, monitoring devices, biopsy and other devices and equipment which may be brought into the magnet room for magnetic field tolerances. Facilities should consider providing physical indications of critical gauss lines in the construction of the magnet room to promote the safe and effective use of MR Conditional equipment, as appropriate.

\section{Infection control (Zone IV)}

Because of safety concerns regarding incidental personnel within the MR suite, restricting housekeeping and cleaning personnel from Zone III and/or IV regions may give rise to concerns regarding the cleanliness of the MR suite. Magnet room finishes and construction details should be designed to facilitate cleaning by appropriately trained staff with nonmotorized equipment. Additionally, as the numbers of MRguided procedures and interventional applications grow, basic infection control protocols, such as seamless floorings, scrub-able surfaces and hand washing stations should be considered.

\section{Limits of applicability/recommended design assistance}

The facility design issues identified in this document address only general safety design issues for MRI 
suites. There are a multitude of site-specific and magnet-specific operational and technical design considerations relevant to MR facility design and construction that are not addressed in these Guidelines. These issues include, but are not limited to, patient acuity, staff access, modality conflicts, vibration sensitivity, throughput/efficiency, HIPAA considerations, magnetic contamination, sound transmission, magnet shim tolerances, shielding design, moving metal interferences, MR equipment upgrades, electromagnetic interference, and many others.

In addition to incorporating the guidance from this document, a facility would be well advised to seek expert assistance in the planning and design of MRI and multi-modal radiology suites.

\section{APPENDIX 4}

\section{MR Facility Emergency Preparedness Guidelines}

Healthcare facilities have a unique obligation to minimize the disruption from disasters and hasten their ability to restore critical patient care services when interrupted.

Those charged with the operation of MRI facilities have the added complexities of protecting not only the staff and structure, but also the equipment which may be extraordinarily sensitive to changes in its environment, including vibration, power supply, and water damage.

In the fall of 2005, many watched as hurricanes Katrina and Rita devastated vast swathes of the United States' Gulf Coast. Those facilities which were well prepared for the damage, loss of power, and other failures of infrastructure fared far better than those that that were not.

Even those not in the likely path of future Gulf hurricanes may have to contend with earthquakes, tornadoes, fires, ice storms, snowstorms, or blackouts, at some point. Particularly those involved in providing patient care should look to how we will provide care at the times when it is most widely and desperately needed. We may find that the facilities, equipment and infrastructure required to provide clinical care have not been adequately protected.

\section{Water Damage:}

Whether from roof-failure, burst pipes, storm surge or rising rivers, every facility has the potential for water damage to equipment and facilities. Damages can range from inconveniences cured by a couple of hours with a wet-dry vacuum, to flooding of equipment electronics. It takes only a small quantity of water in contact with an MRI scanner to incapacitate or destroy the equipment.

To keep leaking roofs, burst pipes or other overhead damage from dousing MRI equipment, it is recommended that facilities prepare by covering gantries and equipment with sturdy plastic, taped in place, when water damage is an anticipated possibility. To keep processors and gradient cabinets from becoming swamped in a flood situation, electronics that can be lifted up off the ground should be moved as far up off of the floor as possible. RF shields, particularly the floor assembly, may be significantly damaged and need to be replaced in a flood situation if not designed to protect against water damage.

During the 2005 hurricanes, many hospitals and imaging facilities that had emergency generators to help restore power discovered that their sites had generators, or other critical supplies, in basements or other low-lying areas that were flooded. Facilities should evaluate risks from water damage and assess their preparations for failure of the building enclosure as well as the potential for a flood situation.

\section{Structural damage:}

MRI presents a particular challenge with structural failure. Though unlikely with current magnet systems, vibrations from seismic events do have the potential to initiate a quench of the magnet system. Structural damage or motion may also damage the RF shield enclosure, potentially degrading image quality until the shield is repaired.

\section{Power Outage:}

Without electrical power to the vacuum pump / cold head to keep the cryogen within a superconducting MRI liquefied, the cryogen will begin to boil off at an accelerated rate. Depending upon cryogen vent design and boil off rate, the additional cryogenic gas discharge may freeze solid any accumulated water in the cryogen vent, occluding the pipe and increasing the possibility for a cryogen vent breach in the event of a quench.

At some point if power to the vacuum pump is not restored, likely a couple days to perhaps a week after power is lost, the magnet will spontaneously quench, discharging most or all of its remaining cryogenic gasses. This poses a safety risk to anyone near the discharge and runs a small but finite risk of potentially permanently damaging the magnet coils.

However, if power to the vacuum pump/cold head and cryogen levels is restored before a quench, there should be no long-term consequences to the magnet's operation from a power interruption.

Temporary electrical power may be provided either through on-site or portable generators. Co-generation, or generating one's own electricity all the time, may not be economically feasible for smaller or standalone sites, but is increasingly appealing to hospitals for several reasons, with emergency capacity being only one.

\section{Quench:}

During the 2005 hurricanes, facilities, fearing extensive damage to their MRI systems from water or protracted power outages, manually initiated pre-emptive quenches. Under the best circumstances, a quench subjects a magnet to a change of $500^{\circ} \mathrm{F}$ thermal shock within a few dozen seconds, which can cause major physical damage. Rarely, it is possible for the venting cryogenic gases to breach the quench tube and cause significant damage to the magnet room and/or 
jeopardize the safety of those in the vicinity. At one New Orleans area facility that elected to preemptively quench its magnets, the quench tube reportedly failed and the pressure from the expanding cryogen blew out the control room radiofrequency window (personal communication, Tobias Gilk, October 2005).

Because of the risks to personnel, equipment and physical facilities, manual magnet quenches are to be initiated only after careful consideration and preparation. In addition to following those specific recommendations provided by the MRI manufacturer, a facility should initiate a pre-emptive quench in nonemergent situations only after verifying the function of emergency exhaust systems, verifying or providing means of pressure relief and a preliminary visual inspection of the cryogen vent pipe as it leaves the MR unit to check for signs of water or ice inside the pipe (including water leaking from fittings or condensation forming on vent pipe sections). If/when feasible, a discussion with the device manufacturer regarding an intentional controlled static magnetic field ramp-down may be advisable.

\section{Fire/Police:}

Though very infrequent, MR suites have been the scene of emergencies requiring fire and/or police response. While it is highly likely that this will be the first time many of the responders have been to an MR suite, this should not be the first time that responding organizations have been introduced to the safety issues for MR. Sites are encouraged to invite police and fire representatives to presentations on MR safety and to provide them with facility tours.

\section{Code:}

In the event that a person within the MR suite should require emergency medical attention, it is imperative that those responding to a call for assistance are aware of, and comply with, MR safety protocols. This includes nurses, physicians, respiratory technicians, paramedics, security, and others.

The impulse to respond immediately must be tempered by an orderly and efficient process to minimize risks to patients, staff and equipment. This requires specialized training for code teams and, as with Fire/ Police responses, clear lines of authority for screening, access restrictions and quench authority. Full resuscitation of patients within Zone IV is complicated by the inability to accurately interpret electrocardiographic data. Furthermore, this may place at risk of injury all within the Zone IV from ferromagnetic objects which may be on, within, or brought into Zone IV by emergency response personnel responding to a code if one is called in that area. Therefore, after initiating basic cardiopulmonary resuscitation (airway, breathing, chest compressions), the patient should be immediately moved out of Zone IV to a prospectively designated location where the code can be run or where the patient will remain until the arrival of emergent response personnel.

It is strongly advised that all MR facilities perform regular drills to rehearse and refine emergency response protocols to protect patients, MR staff and responders.

\section{Prevention:}

While it is the nature of emergencies to be surprises, we can anticipate the types of incidents that have higher likelihoods given our facilities, practices and locations. Every facility can anticipate the potential for flooding, fire and code situations. In addition to these, many areas (California and coastal Alaska, for example) can expect earthquakes. The central and southern plains states of the U.S. can anticipate tornados. Colder climates can expect massive snows or ice storms.

State and federal offices of emergency preparedness are dedicated to anticipating and preparing for the specific threats to your region. These can serve as an excellent resource regarding risks and strategies for preparation.

Once a disaster has struck, it is important to assess what the immediate needs of the community are and to restore those critical patient care services first.

Damage to MRI equipment and facilities may not be repaired as quickly. For gravely incapacitated facilities, semi-trailer based MRI units may be the only means of quickly restoring radiology capacity.

All healthcare facilities should have emergency preparedness plans. The healthcare plans for MRI facilities should specifically address the unique aspects of MRI equipment. These plans should define who has the authority to authorize nonemergent quenches, procedures for emergency or backup power for vacuum pump/cold head, as well as instructions on how to protect gantries and sensitive electronics. Facilities should have the necessary supplies prepositioned and checklists for preparatory and responsive actions. Emergency preparedness plans should also include information necessary for restoring clinical services, including contacts for MRI system vendor, RF shield vendor, cryogen contractor, MR suite architect and construction contractor, local and state officials, and affiliated hospital and professional organizations.

Below are a few questions that may facilitate the development of an emergency preparedness plan specific to the needs of a facility.

- What are the likely/possible natural disasters to affect the area?

- What are the likely/possible man-made disasters to affect the area?

- Is electrical power likely to be interrupted?

- Would other utilities (natural gas, telecommunications, etc.) likely be interrupted?

- What equipment would be inoperative during the emergency?

- What equipment could be damaged by the emergency?

- What equipment should be provided with critical or backup power?

- If the utility service is not quickly restored, what other risks may arise?

- Would patients and staff be able to get to the facility? 
- Would patients or staff be trapped at the facility?

- How critical is each patient care service provided at the facility?

- How does the facility protect the equipment needed to support each service?

- How does the facility protect the patient data (including such options as off site storage) from each service?

- If the facility does not have the resources on site, who can provide them?

\section{REFERENCES}

1. Patient death illustrates the importance of adhering to safety precautions in magnetic resonance environments. Health Devices 2001;30:311-314.

2. Chaljub G, Kramer LA, Johnson RF III, Johnson RF Jr, Singh H, Crow WN. Projectile cylinder accidents resulting from the presence of ferromagnetic nitrous oxide or oxygen tanks in the MR suite. AJR Am J Roentgenol 2001;177:27-30.

3. Kanal E, Borgstede JP, Barkovich AJ, et al. American College of Radiology White Paper on MR Safety: 2004 update and revisions. AJR Am J Roentgenol 2004;182:1111-1114.

4. Kanal E, Borgstede JP, Barkovich AJ, et al. American College of Radiology White Paper on MR Safety. AJR Am J Roentgenol 2002; 178:1335-1347.

5. Kanal E, Shellock FG. Policies, guidelines, and recommendations for MR imaging safety and patient management. SMRI Safety Committee. J Magn Reson Imaging 1992;2:247-248.

6. Shellock FG, Kanal E. Policies, guidelines, and recommendations for MR imaging safety and patient management. SMRI Safety Committee. J Magn Reson Imaging 1991;1:97-101.

7. ACR practice guideline for performing and interpreting magnetic resonance imaging (MRI). Available at: http://www.acr.org/ SecondaryMainMenuCategories/quality_safety/guidelines/dx/mri_ performing_interpreting.aspx. Accessed September 13, 2011.

8. Shellock FG, Kanal E. Guidelines and recommendations for MR imaging safety and patient management. III. Questionnaire for screening patients before MR procedures. The SMRI Safety Committee. J Magn Reson Imaging 1994;4:749-751.

9. Kanal E, Barkovich AJ, Bell C, et al. ACR guidance document for safe MR practices: 2007. AJR Am J Roentgenol 2007;188: 1447-1474.

10. Device Advice: Comprehensive regulatory assistance. Available at: http://www.fda.gov/MedicalDevices/DeviceRegulationandGuidance/ default.htm. Accessed September 19, 2011.

11. Jarvik JG, Ramsey S. Radiographic screening for orbital foreign bodies prior to MR imaging: is it worth it? AJNR Am J Neuroradiol 2000;21:245-247.

12. Seidenwurm DJ, McDonnell CH III, Raghavan N, Breslau J. Cost utility analysis of radiographic screening for an orbital foreign body before MR imaging. AJNR Am J Neuroradiol 2000;21: 426-433.

13. Kanal E, Gillen J, Evans JA, Savitz DA, Shellock FG. Survey of reproductive health among female MR workers. Radiology 1993; 187:395-399.

14. American Academy of Pediatrics Committee on Drugs: Guidelines for monitoring and management of pediatric patients during and after sedation for diagnostic and therapeutic procedures. Pediatrics 1992;89:1110-1115.

15. Guidelines for monitoring and management of pediatric patients during and after sedation for diagnostic and therapeutic procedures: addendum. Pediatrics 2002;110:836-838.

16. Practice guidelines for sedation and analgesia by non-anesthesiologists: An updated report by the American Society of Anesthesiologists Task Force on Sedation and Analgesia by NonAnesthesiologists. Anesthesiology 2002;96:1004-1017.

17. Standards and intents for sedation and anesthesia care. Comprehensive Accreditation Manual for Hospitals. Chicago, IL: Joint Commission on Accreditation of Healthcare Organizations; 2002: Report no. TX 2-2.

18. US Food and Drug Administration Manufacturer and User Facility Device Experience (MAUDE) Data Base (MDR Report Key 434259,
489264, 410913). Available at: http://www.accessdata.fda.gov/ scripts/cdrh/cfdocs/cfMAUDE/TextSearch.cfm. Accessed September $8,2011$.

19. Gandhi OP, Chen XB. Specific absorption rates and induced current densities for an anatomy-based model of the human for exposure to time-varying magnetic fields of MRI. Magn Reson Med 1999;41:816-823.

20. Konings MK, Bartels LW, Smits HF, Bakker CJ. Heating around intravascular guidewires by resonating RF waves. J Magn Reson Imaging 2000;12:79-85.

21. Food and Drug Administration Public Health Advisory: Risk of Burns during MRI Scans from Transdermal Drug Patches with Metallic Backings, March 5, 2009, Updated March 9, 2009. Available at: http://www.fda.gov/Drugs/DrugSafety/PostmarketDrugSafetyInformationforPatientsandProviders/DrugSafetyInforma tionforHeathcareProfessionals/PublicHealthAdvisories/ucm111313. htm. Accessed May 15, 2012.

22. IEC 60601-2-33, Ed. 2.0 Medical Electrical Equipment-Part 2: Particular requirements for the safety of magnetic resonance equipment for medical diagnosis. Geneva, Switzerland: International Electrotechnical Commission (IEC); 2002.

23. ACR-SIR practice guideline for adult sedation/analgesia. Available at: http://www.acr.org/SecondaryMainMenuCategories/quality_safety/guidelines/iv/adult_sedation.aspx. Accessed September 13, 2011.

24. Standards for basic anesthesic monitoring. (Approved by the ASA House of Delegates on October 21, 1986, and last amended on October 20, 2010 with an effective date of July 1, 2011.) Park Ridge, IL: American Society of Anesthesiologists.

25. Standards for postanesthesia care. (Approved by the ASA House of Delegates on October 27, 2004, and last amended on October 21, 2009.) Park Ridge, IL: American Society of Anesthesiologists.

26. Statement on non-operating room anesthetizing locations. (Approved by the ASA House of Delegates on October 19, 1994 and last amended on October 22, 2008.) Park Ridge, IL: American Society of Anesthesiologists.

27. American College of Radiology. ACR digest of council actions. Reston, VA: ACR, 1999:126 (Res 1-H 1987, 1997).

28. American College of Radiology. ACR Manual on Contrast Media, Version 8, 2012. Available at: http://www.acr.org/SecondaryMain MenuCategories/quality_safety/contrast_manual.aspx. Accessed September 13, 2011.

29. Al-Sabagh KH, Christensen BE, Thogersen AM, et al. [Safety of magnetic resonance imaging in patients with pacemaker and implantable defibrillator]. Ugeskr Laeger 2010;172:1740-1744.

30. Gimbel JR. Unexpected asystole during 3T magnetic resonance imaging of a pacemaker-dependent patient with a 'modern' pacemaker. Europace 2009;11:1241-1242.

31. Gotte MJ, Russel IK, de Roest GJ, et al. Magnetic resonance imaging, pacemakers and implantable cardioverter-defibrillators: current situation and clinical perspective. Neth Heart J 2010;18: 31-37.

32. Avery JK. Loss Prevention case of the month. Not my responsibility! J Tenn Med Assoc 1988;81:523.

33. Ferris NJ, Kavnoudias H, Thiel C, Stuckey S. The 2005 Australian MRI safety survey. AJR Am J Roentgenol 2007;188: 1388-1394.

34. Irnich W, Irnich B, Bartsch C, Stertmann WA, Gufler H, Weiler G. Do we need pacemakers resistant to magnetic resonance imaging? Europace 2005;7:353-365.

35. Langman DA, Goldberg IB, Finn JP, Ennis DB. Pacemaker lead tip heating in abandoned and pacemaker-attached leads at 1.5 Tesla MRI. J Magn Reson Imaging 2011;33:426-431.

36. Hartnell GG, Spence L, Hughes LA, Cohen MC, Saouaf R, Buff B. Safety of MR imaging in patients who have retained metallic materials after cardiac surgery. AJR Am J Roentgenol 1997;168: 1157-1159.

37. Murphy KJ, Cohan RH, Ellis JH. MR imaging in patients with epicardial pacemaker wires. AJR Am J Roentgenol 1999;172: 727-728.

38. Kanal E. Safety of MR imaging in patients with retained epicardial pacer wires. AJR Am J Roentgenol 1998;170:213-214.

39. Faris OP, Shein M. Food and Drug Administration perspective: Magnetic resonance imaging of pacemaker and implantable cardioverter-defibrillator patients. Circulation 2006;114:1232-1233. 
40. Gimbel JR. Magnetic resonance imaging of implantable cardiac rhythm devices at 3.0 tesla. Pacing Clin Electrophysiol 2008;31: 795-801.

41. Nazarian S, Halperin HR. How to perform magnetic resonance imaging on patients with implantable cardiac arrhythmia devices. Heart Rhythm 2009;6:138-143.

42. Gimbel JR. Guidelines and the growing service burden. J Interv Card Electrophysiol 2010;28:83-85.

43. Hauser RG, Kallinen L. Deaths associated with implantable cardioverter defibrillator failure and deactivation reported in the United States Food and Drug Administration Manufacturer and
User Facility Device Experience Database. Heart Rhythm 2004;1: 399-405.

44. Jilek C, Tzeis S, Reents $\mathrm{T}$, et al. Safety of implantable pacemakers and cardioverter defibrillators in the magnetic field of a novel remote magnetic navigation system. J Cardiovasc Electrophysiol 2010;21:1136-1141.

45. Gimbel JR. Unexpected pacing inhibition upon exposure to the 3T static magnetic field prior to imaging acquisition: what is the mechanism? Heart Rhythm 2011;8:944-945.

46. The Facility Guidelines Institute. Guidelines for the Design and Construction of Health Care Facilities, 2010 edition. Chicago: American Society for Healthcare Engineering; 2010. 